TRANSACTIONS OF THE

AMERICAN MATHEMATICAL SOCIETY

Volume 356, Number 1, Pages 245-265

S 0002-9947(03)03422-6

Article electronically published on August 26, 2003

\title{
IDEALS OF THE COHOMOLOGY RINGS OF HILBERT SCHEMES AND THEIR APPLICATIONS
}

\author{
WEI-PING LI, ZHENBO QIN, AND WEIQIANG WANG
}

\begin{abstract}
We study the ideals of the rational cohomology ring of the Hilbert scheme $X^{[n]}$ of $n$ points on a smooth projective surface $X$. As an application, for a large class of smooth quasi-projective surfaces $X$, we show that every cup product structure constant of $H^{*}\left(X^{[n]}\right)$ is independent of $n$; moreover, we obtain two sets of ring generators for the cohomology ring $H^{*}\left(X^{[n]}\right)$.

Similar results are established for the Chen-Ruan orbifold cohomology ring of the symmetric product. In particular, we prove a ring isomorphism between $H^{*}\left(X^{[n]} ; \mathbb{C}\right)$ and $H_{\text {orb }}^{*}\left(X^{n} / S_{n} ; \mathbb{C}\right)$ for a large class of smooth quasi-projective surfaces with numerically trivial canonical class.
\end{abstract}

\section{INTRODUCTION}

This is a sequel to LQW1-LQW4 and QW]. We continue the study of the cohomology rings of the Hilbert schemes $X^{[n]}$ of $n$ points on a smooth surface $X$ and the Chen-Ruan orbifold cohomology rings of the symmetric products $X^{n} / S_{n}$. In this paper, the surface $X$ is allowed to be projective as well as quasi-projective (our usage of the terminology "quasi-projective" excludes "projective").

In [Lehn, [LQW1-LQW4, [LS2, which were in turn built on the earlier works Got], [VW], [Na1], Na2], Gro and others, the connections between vertex operators and the multiplicative structure of the rational cohomology group $H^{*}\left(X^{[n]}\right)$ when $X$ is projective have been developed. These connections have been successfully applied to unravel various structures on the cohomology ring of $X^{[n]}$. However, the situation changes dramatically when $X$ is quasi-projective. To date, the understanding of the cohomology ring $H^{*}\left(X^{[n]}\right)$ for $X$ quasi-projective has been rather limited with the exception of the affine plane [ES], [Lehn], [LS1, [Vas] and the minimal resolution of a simple singularity $\mathbb{C}^{2} / \Gamma$, where $\Gamma$ is a finite group of $S L_{2}(\mathbb{C})$ (cf. $[\mathrm{Wa}]$ ).

Besides the minimal resolutions mentioned above, typical important examples of quasi-projective surfaces include the cotangent bundle of a smooth projective curve and the surface obtained from a smooth projective surface by deleting a point. All these surfaces are among a class of quasi-projective surfaces which satisfy what we call the S-property (see Definition 4.2). One of the goals of the present paper is to establish some general results of the cohomology rings of the Hilbert schemes

Received by the editors July 5, 2002.

2000 Mathematics Subject Classification. Primary 14C05; Secondary 14F25, 17B69.

Key words and phrases. Heisenberg algebra, Hilbert scheme, cohomology ring.

The first author was partially supported by the grant HKUST6170/99P.

The second author was partially supported by an NSF grant.

The third author was partially supported by an NSF grant. 
of points for such a large class of quasi-projective surfaces. These results are in general not valid for projective surfaces, and conjecturally, they hold for every quasi-projective surface (without the S-property assumption).

We begin with a study of the ideals $\mathcal{I}^{[n]}$ of the cohomology ring $H^{*}\left(X^{[n]}\right)$ for a smooth projective surface $X$, which are induced from ideals $\mathcal{I}$ of the cohomology ring of $X$ (see Definition [2.6). We prove that part of the cup product structure constants for the cohomology rings of the Hilbert schemes $X^{[n]}$ with respect to Nakajima-Grojnowski's Heisenberg monomial linear basis is independent of $n$. This part of the cup product structure constants can be regarded as coming from the complement to the ideal $\mathcal{I}^{[n]}$. The methods used in establishing the above results follow the techniques developed in LQW3, [LQW4.

While the results on the ideals $\mathcal{I}^{[n]}$ are of independent interest, they are developed with applications to smooth quasi-projective surfaces in mind. As the first application, we prove the following result (Theorem 4.3).

Theorem 1.1. Let $X$ be a smooth quasi-projective surface with the S-property. Then, all the cup product structure constants of $H^{*}\left(X^{[n]}\right)$ are independent of $n$.

The precise definition of the structure constants is given by (4.3). The $n$ independence has been conjectured in Wa to be true for every smooth quasiprojective surface, and has its counterpart in the framework of the class algebras of the wreath products. It allows us to introduce a universal ring $\mathcal{G}_{X}$ for a smooth quasi-projective surface $X$ with the S-property, which governs the cohomology rings $H^{*}\left(X^{[n]}\right)$ for a fixed $X$ and all $n$. This ring $\mathcal{G}_{X}$ will be called the FH ring associated to $X$, as it is analogous to the one arising in the framework of symmetric groups and wreath products $[\mathrm{FH}]$, Wa]. We further determine the ring structure of $\mathcal{G}_{X}$, and obtain two sets of ring generators of $H^{*}\left(X^{[n]}\right)$ which are the quasi-projective counterparts of the main results in LQW1, [LQW2. We remark that a universal ring termed as the Hilbert ring was introduced in [LQW3 which governs the cohomology rings $H^{*}\left(X^{[n]}\right)$ for a fixed projective $X$ and all $n$. These two universal rings reflect distinct structures of the corresponding cohomology rings.

Observe that a distinguished Heisenberg generator (i.e. the first annihilation operator $) \mathfrak{a}_{1}\left([x]_{c}\right)$ is cohomology degree-preserving, where $[x]_{c} \in H_{c}^{4}(X)$ denotes the Poincaré dual of the homology class in $H_{0}(X)$ represented by a point in $X$. It turns out that Theorem 1.1 admits the following equivalent reformulation.

Corollary 1.2. Let $X$ be a smooth quasi-projective surface with the S-property. Then $-\mathfrak{a}_{1}\left([x]_{c}\right): H^{*}\left(X^{[n+1]}\right) \rightarrow H^{*}\left(X^{[n]}\right)$ is a surjective ring homomorphism.

Recall a well-known fact that the Hilbert-Chow morphism from the Hilbert schemes $X^{[n]}$ to the symmetric product $X^{n} / S_{n}$ is a resolution of singularity. In $\mathrm{CR}$, Chen and Ruan introduced the notion of the orbifold cohomology ring $H_{\mathrm{orb}}^{*}(Y)$ for an orbifold $Y$. Following the general machinery developed in [QW], we automatically establish results parallel to those stated in the previous paragraphs for the orbifold cohomology rings $H_{\mathrm{orb}}^{*}\left(X^{n} / S_{n}\right)$ of the symmetric products $X^{n} / S_{n}$. We further prove the following result (Theorem 5.4 and Remark [5.6).

Theorem 1.3. Let $X$ be a smooth quasi-projective surface with the $S$-property and a numerically trivial canonical class. Then, the cohomology $\operatorname{ring} H^{*}\left(X^{[n]} ; \mathbb{C}\right)$ is isomorphic to the orbifold cohomology ring $H_{\text {orb }}^{*}\left(X^{n} / S_{n} ; \mathbb{C}\right)$. 
A conjecture of Ruan [Ru1, [Ru2] states that the cohomology ring $H^{*}(Z ; \mathbb{C})$ with $\mathbb{C}$-coefficient is isomorphic to the orbifold cohomology $\operatorname{ring} H_{\text {orb }}^{*}(Y ; \mathbb{C})$ with $\mathbb{C}$-coefficient for any hyperkahler resolution $Z$ of an orbifold $Y$. In light of this conjecture, we obtain a very interesting question: for which surfaces satisfying the assumption in Theorem 1.3 do the corresponding Hilbert schemes of points carry a hyperkahler structure (and Theorem 1.3 for these surfaces confirms Ruan's conjecture)? For example, the Hilbert scheme of points on the minimal resolution of a simple singularity carries a hyperkahler structure $\mathrm{Na2}$. We remark that Theorem 1.3 holds when the surface $X$ is the cotangent bundle of a smooth projective curve or the minimal resolution of a simple singularity $\mathbb{C}^{2} / \Gamma$. In the special case when $X$ is the affine plane (i.e. $\Gamma$ is trivial), we recover the main results of [LS1], Vas (also cf. [Ru1]). An isomorphism as in Theorem 1.3 when $X$ is projective with a numerically trivial canonical class was earlier established by various authors [LS2], [FG], Uri], QW], which also supported Ruan's conjecture.

The paper is organized as follows. In Section 2 we study the ideals in $H^{*}\left(X^{[n]}\right)$ for a smooth projective surface $X$. In Section 3 we verify the partial $n$-independence of the cup product structure constants for the cohomology rings of $X^{[n]}$ when $X$ is a smooth projective surface. In Section 4 we prove Theorem 1.1 and construct as well as study the FH ring $\mathcal{G}_{X}$. In Section 5 , for a smooth quasi-projective surface $X$ with the S-property, we formulate the analogous results for the orbifold cohomology ring of the symmetric product $X^{n} / S_{n}$. Furthermore, we establish Theorem 1.3

Conventions. All cohomology groups are in $\mathbb{Q}$-coefficients unless otherwise indicated. The cup product of two cohomology classes $\alpha$ and $\beta$ is denoted by $\alpha \cdot \beta$ or simply by $\alpha \beta$. For a continuous map $p: Y_{1} \rightarrow Y_{2}$ between two smooth compact manifolds and for $\alpha_{1} \in H^{*}\left(Y_{1}\right)$, let $p_{*}\left(\alpha_{1}\right)=\mathrm{PD}^{-1} p_{*}\left(\mathrm{PD}\left(\alpha_{1}\right)\right)$, where $\mathrm{PD}$ stands for the Poincaré duality. For a smooth projective surface $X$, let $1_{X} \in H^{0}(X)$ be the fundamental cohomology class of $X$, and let $[x] \in H^{4}(X)$ be the Poincaré dual of the homology class represented by a point $x \in X$.

\section{Ideals in $H^{*}\left(X^{[n]}\right)$ FOR $X$ Projective}

2.1. Preliminaries. Let $X$ be a smooth projective complex surface with the canonical class $K$ and the Euler class $e$, and let $X^{[n]}$ be the Hilbert scheme of points in $X$. It is known that $X^{[n]}$ is a desingularization of the symmetric product $X^{n} / S_{n}$. Let $\mathcal{Z}_{n} \subset X^{[n]} \times X$ be the universal subscheme, and $\mathbb{H}_{X}=\bigoplus_{n=0}^{\infty} H^{*}\left(X^{[n]}\right)$. Recall that a Heisenberg algebra was defined in [Na2] acting on $\mathbb{H}_{X}$. In this paper we follow the notations and conventions in [LQW3], LQW4, for the Heisenberg algebra generators however. Namely, we have the operators $\mathfrak{a}_{n}(\alpha) \in \operatorname{End}\left(\mathbb{H}_{X}\right)$ with $\alpha \in H^{*}(X)$ and $n \in \mathbb{Z}$ which satisfy the following Heisenberg algebra commutation relation:

$$
\left[\mathfrak{a}_{m}(\alpha), \mathfrak{a}_{n}(\beta)\right]=-m \delta_{m,-n} \int_{X}(\alpha \beta) \cdot \operatorname{Id}_{\mathbb{H}_{X}} \cdot
$$

Here and throughout the paper, the Lie brackets are understood in the super sense according to the parity of the cohomology degrees of the cohomology classes involved. When $n>0$, we often refer to $\mathfrak{a}_{-n}(\alpha)$ (resp. $\mathfrak{a}_{n}(\alpha)$ ) as the creation (resp. annihilation) operator. The space $\mathbb{H}_{X}$ is an irreducible module over the 
Heisenberg algebra generated by the operators $\mathfrak{a}_{n}(\alpha)$ with a highest weight vector $|0\rangle=1 \in H^{0}\left(X^{[0]}\right) \cong \mathbb{Q}$. It follows that $\mathbb{H}_{X}$ is linearly spanned by all the Heisenberg monomials $\mathfrak{a}_{-n_{1}}\left(\alpha_{1}\right) \cdots \mathfrak{a}_{-n_{k}}\left(\alpha_{k}\right)|0\rangle$, where $k \geq 0$ and $n_{1}, \ldots, n_{k}>0$.

For $n>0$ and a homogeneous class $\gamma \in H^{*}(X)$, let $|\gamma|=s$ if $\gamma \in H^{s}(X)$, and let $G_{i}(\gamma, n)$ be the homogeneous component in $H^{|\gamma|+2 i}\left(X^{[n]}\right)$ of

$$
G(\gamma, n)=p_{1 *}\left(\operatorname{ch}\left(\mathcal{O}_{\mathcal{Z}_{n}}\right) \cdot p_{2}^{*} \operatorname{td}(X) \cdot p_{2}^{*} \gamma\right) \in H^{*}\left(X^{[n]}\right)
$$

where $p_{1}$ and $p_{2}$ are the projections of $X^{[n]} \times X$ to $X^{[n]}$ and $X$, respectively. We extend the notion $G_{i}(\gamma, n)$ linearly to an arbitrary $\gamma \in H^{*}(X)$, and set $G(\gamma, 0)=0$. The Chern character operator $\mathfrak{G}_{i}(\gamma) \in \operatorname{End}\left(\mathbb{H}_{X}\right)$ is defined to be the operator acting on the component $H^{*}\left(X^{[n]}\right)$ by the cup product with $G_{i}(\gamma, n)$. It was proved in LQW1 that the cohomology ring of $X^{[n]}$ is generated by the classes $G_{i}(\gamma, n)$, where $0 \leq i<n$ and $\gamma$ runs over a linear basis of $H^{*}(X)$.

For $k \geq 1$, let $\tau_{k *}: H^{*}(X) \rightarrow H^{*}\left(X^{k}\right)$ be the map induced by the diagonal embedding $\tau_{k}: X \rightarrow X^{k}$, and let $\mathfrak{a}_{m_{1}} \cdots \mathfrak{a}_{m_{k}}\left(\tau_{k *}(\alpha)\right)$ denote $\sum_{j} \mathfrak{a}_{m_{1}}\left(\alpha_{j, 1}\right) \cdots \mathfrak{a}_{m_{k}}\left(\alpha_{j, k}\right)$ when $\tau_{k *} \alpha=\sum_{j} \alpha_{j, 1} \otimes \cdots \otimes \alpha_{j, k}$ via the Künneth decomposition of $H^{*}\left(X^{k}\right)$.

The following two lemmas were proved in LQW3, where $\tau_{0 *}(\alpha)$ denotes $\int_{X} \alpha$.

Lemma 2.1. Let $k, s \geq 1, n_{1}, \ldots, n_{k}, m_{1}, \ldots, m_{s} \in \mathbb{Z}$, and $\alpha, \beta \in H^{*}(X)$. Then

(i) the commutator $\left[\mathfrak{a}_{n_{1}} \cdots \mathfrak{a}_{n_{k}}\left(\tau_{k *} \alpha\right), \mathfrak{a}_{m_{1}} \cdots \mathfrak{a}_{m_{s}}\left(\tau_{s *} \beta\right)\right]$ is equal to

$$
-\sum_{t=1}^{k} \sum_{j=1}^{s} n_{t} \delta_{n_{t},-m_{j}} \cdot\left(\prod_{\ell=1}^{j-1} \mathfrak{a}_{m_{\ell}} \prod_{1 \leq u \leq k, u \neq t} \mathfrak{a}_{n_{u}} \prod_{\ell=j+1}^{s} \mathfrak{a}_{m_{\ell}}\right)\left(\tau_{(k+s-2) *}(\alpha \beta)\right)
$$

(ii) let $j$ satisfy $1 \leq j<k$. Then, $\mathfrak{a}_{n_{1}} \cdots \mathfrak{a}_{n_{k}}\left(\tau_{k *} \alpha\right)$ is equal to

$$
\left(\prod_{1 \leq s<j} \mathfrak{a}_{n_{s}} \cdot \mathfrak{a}_{n_{j+1}} \mathfrak{a}_{n_{j}} \cdot \prod_{j+1<s \leq k} \mathfrak{a}_{n_{s}}\right)\left(\tau_{k *} \alpha\right)-n_{j} \delta_{n_{j},-n_{j+1}} \prod_{\substack{1 \leq s \leq k \\ s \neq j, j+1}} \mathfrak{a}_{n_{s}}\left(\tau_{(k-2) *}(e \alpha)\right) .
$$

Lemma 2.2. Fix $k \geq 0$ and $b \geq 1$. Let $\mathfrak{g} \in \operatorname{End}(\mathbb{H})$ be of bi-degree $(\tilde{s}, s)$ satisfying

$$
\left[\left[\cdots\left[\mathfrak{g}, \mathfrak{a}_{m_{1}}\left(\beta_{1}\right)\right], \cdots\right], \mathfrak{a}_{m_{k+2}}\left(\beta_{k+2}\right)\right]=0
$$

whenever $m_{i}<0$ and $\beta_{i} \in H^{*}(X)$ for each $i$. Let $A=\mathfrak{a}_{-n_{1}}\left(\alpha_{1}\right) \cdots \mathfrak{a}_{-n_{b}}\left(\alpha_{b}\right)|0\rangle$ where $n_{1}, \ldots, n_{b}>0$ and $\alpha_{1}, \ldots, \alpha_{b} \in H^{*}(X)$. Then, $\mathfrak{g}(A)$ is equal to

$$
\begin{aligned}
& \sum_{i=0}^{k+1} \sum_{\sigma_{i}}(-1)^{s \sum_{\ell \in \sigma_{i}^{0}}\left|\alpha_{\ell}\right|+\sum_{j=1}^{i} \sum_{\ell \in \sigma_{i}^{0}, \ell>\sigma_{i}(j)}\left|\alpha_{\sigma_{i}(j)}\right|\left|\alpha_{\ell}\right|} \\
& \cdot\left(\prod_{\ell \in \sigma_{i}^{0}} \mathfrak{a}_{-n_{\ell}}\left(\alpha_{\ell}\right)\right)\left[\left[\cdots\left[\mathfrak{g}, \mathfrak{a}_{-n_{\sigma_{i}(1)}}\left(\alpha_{\sigma_{i}(1)}\right)\right], \cdots\right], \mathfrak{a}_{-n_{\sigma_{i}(i)}}\left(\alpha_{\sigma_{i}(i)}\right)\right]|0\rangle
\end{aligned}
$$

where $0 \leq i \leq k+1, \sigma_{i}$ runs over all the maps $\{1, \ldots, i\} \rightarrow\{1, \ldots, b\}$ satisfying $\sigma_{i}(1)<\cdots<\sigma_{i}(i)$, and $\sigma_{i}^{0}=\left\{\ell \mid 1 \leq \ell \leq b, \ell \neq \sigma_{i}(1), \ldots, \sigma_{i}(i)\right\}$.

Definition 2.3. Let $X$ be a smooth projective surface.

(i) Let $\alpha \in H^{*}(X)$, and $\lambda=\left(\cdots(-2)^{m_{-2}}(-1)^{m_{-1}} 1^{m_{1}} 2^{m_{2}} \cdots\right)$ be a generalized partition of the integer $n=\sum_{i} i m_{i}$ whose part $i \in \mathbb{Z}$ has multiplicity $m_{i}$. 
Define $\ell(\lambda)=\sum_{i} m_{i},|\lambda|=\sum_{i} i m_{i}=n, s(\lambda)=\sum_{i} i^{2} m_{i}, \lambda^{!}=\prod_{i} m_{i} !$, and

$$
\mathfrak{a}_{\lambda}\left(\tau_{*} \alpha\right)=\left(\prod_{i}\left(\mathfrak{a}_{i}\right)^{m_{i}}\right)\left(\tau_{\ell(\lambda) *} \alpha\right),
$$

where the product $\prod_{i}\left(\mathfrak{a}_{i}\right)^{m_{i}}$ is understood to be $\cdots \mathfrak{a}_{-2}^{m_{-2}} \mathfrak{a}_{-1}^{m_{-1}} \mathfrak{a}_{1}^{m_{1}} \mathfrak{a}_{2}^{m_{2}} \cdots$. Let $-\lambda$ be the generalized partition whose multiplicity of $i \in \mathbb{Z}$ is $m_{-i}$.

(ii) A generalized partition becomes a partition in the usual sense if the multiplicity $m_{i}=0$ for every $i<0$. A partition $\lambda$ of $n$ is denoted by $\lambda \vdash n$.

(iii) We let $\mathbf{1}_{-n}$ denote $\mathfrak{a}_{-1}\left(1_{X}\right)^{n} / n$ ! when $n \geq 0$ and 0 when $n<0$.

When $n \geq 0, \mathbf{1}_{-n}|0\rangle$ is the fundamental cohomology class of the Hilbert scheme $X^{[n]}$. The following theorem was one of the main results proved in LQW4].

Theorem 2.4. Let $k \geq 0$ and $\alpha \in H^{*}(X)$. Then, $\mathfrak{G}_{k}(\alpha)$ is equal to

$$
\begin{gathered}
-\sum_{\ell(\lambda)=k+2,|\lambda|=0} \frac{1}{\lambda^{!}} \mathfrak{a}_{\lambda}\left(\tau_{*} \alpha\right)+\sum_{\ell(\lambda)=k,|\lambda|=0} \frac{s(\lambda)-2}{24 \lambda^{!}} \mathfrak{a}_{\lambda}\left(\tau_{*}(e \alpha)\right) \\
+\sum_{\epsilon \in\left\{K, K^{2}\right\}} \ell(\lambda)=k+2-|\epsilon| / 2,|\lambda|=0 \\
\frac{g_{\epsilon}(\lambda)}{\lambda^{!}} \mathfrak{a}_{\lambda}\left(\tau_{*}(\epsilon \alpha)\right)
\end{gathered}
$$

where all the numbers $g_{\epsilon}(\lambda)$ are independent of $X$ and $\alpha$.

\subsection{Ideals in $H^{*}\left(X^{[n]}\right)$ for $X$ projective.}

Lemma 2.5. Let $\mathcal{I}$ be an ideal in the cohomology ring $H^{*}(X)$. Let $\alpha \in \mathcal{I}$ and $k \geq 2$. Then, the pushforward $\tau_{k *} \alpha$ can be written as $\sum_{j} \alpha_{j, 1} \otimes \cdots \otimes \alpha_{j, k} \in H^{*}\left(X^{k}\right)$ such that for each fixed $j$, there exists some $\ell$ with $\alpha_{j, \ell} \in \mathcal{I}$.

Proof. First of all, note that if $\tau_{k *} \alpha=\sum_{j} \alpha_{j, 1} \otimes \alpha_{j, 2} \otimes \cdots \otimes \alpha_{j, k}$, then $\tau_{(k+1) *} \alpha=$ $\sum_{j}\left(\tau_{2 *} \alpha_{j, 1}\right) \otimes \alpha_{j, 2} \otimes \cdots \otimes \alpha_{j, k}$. Therefore, by induction, it suffices to prove the lemma for $k=2$. Now the case $k=2$ follows from the observation that if we write $\tau_{2 *}\left(1_{X}\right)=\sum_{i} \alpha_{i} \otimes \beta_{i}$, then $\tau_{2 *}(\alpha)=\sum_{i}\left(\alpha \alpha_{i}\right) \otimes \beta_{i}$ with $\left(\alpha \alpha_{i}\right) \in \mathcal{I}$.

In view of the preceding lemma, we introduce the following important definition.

Definition 2.6. Let $X$ be a smooth projective surface, and let $\mathcal{I}$ be an ideal in the cohomology ring $H^{*}(X)$. For $n \geq 1$, define $\mathcal{I}^{[n]}$ to be the subset of $H^{*}\left(X^{[n]}\right)$ consisting of the linear spans of Heisenberg monomials of the form $\mathfrak{a}_{-n_{1}}\left(\alpha_{1}\right) \cdots \mathfrak{a}_{-n_{b}}\left(\alpha_{b}\right)|0\rangle$ where $\alpha_{i} \in \mathcal{I}$ for some $i$, and $n_{1}, \ldots, n_{b}$ are positive with $\sum_{\ell} n_{\ell}=n$.

Lemma 2.7. Let $\mathcal{I}$ be an ideal in the cohomology ring $H^{*}(X)$. Then,

(i) the linear subspace $\mathcal{I}^{[n]}$ in $H^{*}\left(X^{[n]}\right)$ is an ideal, and

(ii) $G_{k}(\alpha, n) \in \mathcal{I}^{[n]}$ if $\alpha \in \mathcal{I}$.

Proof. (i) Recall from [LQW1 that the cohomology $\operatorname{ring} H^{*}\left(X^{[n]}\right)$ is generated by the cohomology classes $G_{k}(\alpha, n)$. So it suffices to prove

$$
\mathfrak{G}_{k}(\alpha) \mathfrak{a}_{-n_{1}}\left(\alpha_{1}\right) \cdots \mathfrak{a}_{-n_{b}}\left(\alpha_{b}\right)|0\rangle \in \mathcal{I}^{[n]}
$$

whenever $\alpha_{1} \in \mathcal{I}$, and $n_{1}, \ldots, n_{b}$ are positive with $\sum_{\ell} n_{\ell}=n$. For simplicity, put $\mathfrak{g}=\mathfrak{G}_{k}(\alpha)$. Then, the operator $\mathfrak{g}$ is of bi-degree $(0,2 k+|\alpha|)$, and satisfies (2.2) by 
Theorem[2.4. Now we see from Lemma 2.2 that $\mathfrak{G}_{k}(\alpha) \mathfrak{a}_{-n_{1}}\left(\alpha_{1}\right) \cdots \mathfrak{a}_{-n_{b}}\left(\alpha_{b}\right)|0\rangle$ is a linear combination of expressions of the form

$$
\left(\prod_{\ell \in \sigma_{i}^{0}} \mathfrak{a}_{-n_{\ell}}\left(\alpha_{\ell}\right)\right)\left[\left[\cdots\left[\mathfrak{g}, \mathfrak{a}_{-n_{\sigma_{i}(1)}}\left(\alpha_{\sigma_{i}(1)}\right)\right], \cdots\right], \mathfrak{a}_{-n_{\sigma_{i}(i)}}\left(\alpha_{\sigma_{i}(i)}\right)\right]|0\rangle,
$$

where $0 \leq i \leq k+1, \sigma_{i}$ maps the set $\{1, \ldots, i\}$ to the set $\{1, \ldots, b\}$ with $\sigma_{i}(1)<$ $\cdots<\sigma_{i}(i)$, and $\sigma_{i}^{0}=\left\{\ell \mid 1 \leq \ell \leq b, \ell \neq \sigma_{i}(1), \ldots, \sigma_{i}(i)\right\}$.

If $1 \in \sigma_{i}^{0}$, then (2.4) is contained in $\mathcal{I}^{[n]}$. In the following, we assume $1 \notin \sigma_{i}^{0}$. So $1=\sigma_{i}(1)$ since $\sigma_{i}(1)<\cdots<\sigma_{i}(i)$. By Theorem 2.4 and Lemma 2.1(i), (2.4) is a linear combination of expressions of the form

$$
\left(\prod_{\ell \in \sigma_{i}^{0}} \mathfrak{a}_{-n_{\ell}}\left(\alpha_{\ell}\right)\right) \mathfrak{a}_{-\lambda}\left(\tau_{*}\left(\epsilon \alpha \alpha_{\sigma_{i}(1)} \cdots \alpha_{\sigma_{i}(i)}\right)\right)|0\rangle
$$

where $\epsilon \in\left\{1_{X}, e, K, K^{2}\right\}, \lambda \vdash \sum_{j=1}^{i} n_{\sigma_{i}(j)}$ and $\ell(\lambda)=k+2-|\epsilon| / 2-i$. By Lemma 2.5, the expression 2.5) is contained in $\mathcal{I}^{[n]}$ since $\alpha_{\sigma_{i}(1)}=\alpha_{1} \in \mathcal{I}$. It follows that (2.4) is contained in $\mathcal{I}^{[n]}$. This proves (2.3).

(ii) Recall from Corollary 4.8 in [LW4] that $G_{k}(\alpha, n)$ is equal to

$$
\begin{aligned}
& \sum_{0 \leq j \leq k} \sum_{\substack{\lambda \vdash(j+1) \\
\ell(\lambda)=k-j+1}} \frac{(-1)^{|\lambda|-1}}{\lambda^{!} \cdot|\lambda| !} \cdot \mathbf{1}_{-(n-j-1)} \mathfrak{a}_{-\lambda}\left(\tau_{*} \alpha\right)|0\rangle \\
& \quad+\sum_{\substack { 0 \leq j \leq k \\
\begin{subarray}{c}{\lambda+(\lambda)=k-j-1 \\
\ell(j+1){ 0 \leq j \leq k \\
\begin{subarray} { c } { \lambda + ( \lambda ) = k - j - 1 \\
\ell ( j + 1 ) } }\end{subarray}} \frac{(-1)^{|\lambda|}}{\lambda^{!} \cdot|\lambda| !} \cdot \frac{|\lambda|+s(\lambda)-2}{24} \cdot \mathbf{1}_{-(n-j-1)} \mathfrak{a}_{-\lambda}\left(\tau_{*}(e \alpha)\right)|0\rangle \\
& \quad+\sum_{\substack{\epsilon \in\left\{K, K^{2}\right\} \\
0 \leq j \leq k}} \sum_{\substack{\lambda \vdash(j+1) \\
\ell(\lambda)=k-j+1-|\epsilon| / 2}} \frac{(-1)^{|\lambda|} g_{\epsilon}\left(\lambda+\left(1^{j+1}\right)\right)}{\lambda^{!} \cdot|\lambda| !} \cdot \mathbf{1}_{-(n-j-1)} \mathfrak{a}_{-\lambda}\left(\tau_{*}(\epsilon \alpha)\right)|0\rangle,
\end{aligned}
$$

where $g_{\epsilon}$ is from Theorem 2.4 and $\lambda+\left(1^{j+1}\right)$ is the partition obtained from $\lambda$ by adding $(j+1)$ to the multiplicity of 1 . So $G_{k}(\alpha, n) \in \mathcal{I}^{[n]}$ if $\alpha \in \mathcal{I}$.

The following technical definition will be used throughout the paper.

Definition 2.8. Let $X$ be a smooth projective surface, $s \geq 1$, and $t_{1}, \ldots, t_{s} \geq 1$. Fix $m_{i, j} \geq 0$ and $\beta_{i, j} \in H^{*}(X)$ for $1 \leq i \leq s$ and $1 \leq j \leq t_{i}$. Then, a universal linear combination of $\prod_{j=1}^{t_{i}} G_{m_{i, j}}\left(\beta_{i, j}, n\right), 1 \leq i \leq s$, is a linear combination of the form $\sum_{i=1}^{s} f_{i} \prod_{j=1}^{t_{i}} G_{m_{i, j}}\left(\beta_{i, j}, n\right)$, where the coefficients $f_{i}$ are independent of $X$ and $n$. A universal linear combination of $\mathfrak{a}_{-n_{i, 1}}\left(\beta_{i, 1}\right) \cdots \mathfrak{a}_{-n_{i, t_{i}}}\left(\beta_{i, t_{i}}\right)|0\rangle, 1 \leq i \leq s$, with $n_{i, j} \geq 1$ and $n_{i, 1}+\ldots+n_{i, t_{i}}=n$ is defined in a similar way.

Theorem 2.9. Let $X$ be a smooth projective surface, and let $\mathcal{I}$ be an ideal in the cohomology ring $H^{*}(X)$. If $\mathcal{I}$ is homogeneous (i.e., $\mathcal{I}=\bigoplus_{i=0}^{4}\left(\mathcal{I} \cap H^{i}(X)\right)$ ), then the ideal $\mathcal{I}^{[n]}$ is generated by the classes $G_{k}(\alpha, n)$ with $\alpha \in \mathcal{I}$. 
Proof. Note that every Heisenberg monomial in the ideal $\mathcal{I}^{[n]}$ can be written as $A=\mathbf{1}_{-\left(n-n_{0}\right)}\left(\prod_{i=1}^{s} \mathfrak{a}_{-n_{i}}\left(\alpha_{i}\right)\right)|0\rangle$, where $s \geq 1, n_{1}, \ldots, n_{s} \geq 1, n_{0}=\sum_{i=1}^{s} n_{i}$, and $\alpha_{\ell}$ is contained in $\mathcal{I}$ and homogeneous for some $\ell$. By Lemma 2.7(ii), it suffices to show that $A \in \mathcal{I}^{[n]}$ is a universal finite linear combination of expressions of the form

$$
\prod_{j=1}^{t} G_{m_{j}}\left(\beta_{j}, n\right)
$$

where $\sum_{j=1}^{t}\left(m_{j}+1\right) \leq n_{0}$, and $\beta_{\ell} \in \mathcal{I}$ for some $\ell$.

Use induction on $n_{0}$. When $n_{0}=1, s=n_{1}=1$. So $A=\mathbf{1}_{-(n-1)} \mathfrak{a}_{-1}\left(\alpha_{1}\right)|0\rangle=$ $G_{0}\left(\alpha_{1}, n\right)$ by (2.6). Hence the statement in the previous paragraph holds for $n_{0}=1$.

Next assume $n_{0}>1$. Let $k_{i}=n_{i}-1$ for $1 \leq i \leq s$. Then, $k_{i} \geq 0$ for every $i$. By the Theorem 4.1 and Lemma 5.1 in LQW3, the cup product $\prod_{i=1}^{s} G_{k_{i}}\left(\alpha_{i}, n\right)$ is equal to $\left(\prod_{i=1}^{s} \frac{(-1)^{k_{i}}}{\left(k_{i}+1\right) !}\right) A$ (defined to be the leading term) plus a universal finite linear combination of expressions $\mathbf{1}_{-\left(n-\tilde{n}_{0}\right)}\left(\prod_{i=1}^{\tilde{s}} \mathfrak{a}_{-\tilde{n}_{i}}\left(\tilde{\alpha}_{i}\right)\right)|0\rangle$, where $\tilde{\alpha}_{\ell} \in \mathcal{I}$ for some $\ell$, $\tilde{s} \geq 1, \tilde{n}_{1}, \ldots, \tilde{n}_{\tilde{s}} \geq 1$, and $\sum_{i=1}^{\tilde{s}} \tilde{n}_{i}=\tilde{n}_{0}<\sum_{i=1}^{s}\left(k_{i}+1\right)=n_{0}$. By induction, $A$ is a universal finite linear combination of expressions of the form (2.7).

Remark 2.10. The assumption in Theorem 2.9 that the ideal $\mathcal{I} \subset H^{*}(X)$ is homogeneous can be dropped when the surface $X$ is simply connected.

2.3. Relation with the affine plane. In the following, we study the quotient ring $H^{*}\left(X^{[n]}\right) / \mathcal{I}^{[n]}$ when $\mathcal{I}=\bigoplus_{\ell=1}^{4} H^{\ell}(X)$. Note that $H^{*}\left(X^{[n]}\right) / \mathcal{I}^{[n]}$ has a linear basis consisting of Heisenberg monomials of the form $\mathfrak{a}_{-n_{1}}\left(1_{X}\right)^{r_{1}} \cdots \mathfrak{a}_{-n_{k}}\left(1_{X}\right)^{r_{k}}|0\rangle$, where $r_{1}, \ldots, r_{k} \geq 1$, and $0<n_{1}<\ldots<n_{k}$ with $\sum_{\ell=1}^{k} r_{\ell} n_{\ell}=n$. So we have an isomorphism of vector spaces

$$
\Phi: \bigoplus_{n \geq 0} H^{*}\left(X^{[n]}\right) / \mathcal{I}^{[n]} \rightarrow \mathbb{Q}\left[q_{1}, q_{2}, \ldots\right],
$$

where $\mathbb{Q}\left[q_{1}, q_{2}, \ldots\right]$ is the polynomial ring in countably infinitely many variables. Setting the degree of the variable $q_{i}$ to be $i$, we see that $\Phi$ maps $H^{*}\left(X^{[n]}\right) / \mathcal{I}^{[n]}$ to the homogeneous component of $\mathbb{Q}\left[q_{1}, q_{2}, \ldots\right]$ of degree $n$.

Lemma 2.11. Let $\mathcal{I}=\bigoplus_{\ell=1}^{4} H^{\ell}(X)$. Then, the quotient ring $H^{*}\left(X^{[n]}\right) / \mathcal{I}^{[n]}$ is generated by the classes $G_{k}\left(1_{X}, n\right), k=0,1, \ldots, n-1$. Moreover,

$$
G_{k}\left(1_{X}, n\right) \equiv \frac{(-1)^{k}}{(k+1) !} \cdot \mathbf{1}_{-(n-k-1)} \mathfrak{a}_{-(k+1)}\left(1_{X}\right)|0\rangle \quad\left(\bmod \mathcal{I}^{[n]}\right) .
$$

Proof. Since the cohomology ring $H^{*}\left(X^{[n]}\right)$ is generated by the classes $G_{k}(\alpha, n)$ with $0 \leq k<n$ and $\alpha \in H^{*}(X)$, the first statement follows from Lemma 2.7(ii). To prove (2.9), we note from (2.6) that the leading term in $G_{k}\left(1_{X}, n\right)$ is $\frac{(-1)^{k}}{(k+1) !}$. $\mathbf{1}_{-(n-k-1)} \mathfrak{a}_{-(k+1)}(\alpha)|0\rangle$ corresponding to $j=k, \lambda \vdash(j+1)=k+1$ and $\ell(\lambda)=$ 
$k-j+1=1$. The other terms in $G_{k}\left(1_{X}, n\right)$ contain $\tau_{i *}(\epsilon)$ with either $i \geq 2$ or $\epsilon=e, K, K^{2} \in \mathcal{I}$, and hence are contained in $\mathcal{I}^{[n]}$. This proves (2.9).

Theorem 2.12. Let $X$ be a smooth projective surface, and $\mathcal{I}=\bigoplus_{\ell=1}^{4} H^{\ell}(X)$. Then, the quotient $H^{*}\left(X^{[n]}\right) / \mathcal{I}^{[n]}$ is isomorphic to the cohomology ring $H^{*}\left(\left(\mathbb{C}^{2}\right)^{[n]}\right)$.

Proof. By Lemma 2.11, the quotient ring $H^{*}\left(X^{[n]}\right) / \mathcal{I}^{[n]}$ is generated by the classes $G_{k}\left(1_{X}, n\right), k=0,1, \ldots, n-1$. So by Theorem 4.10 in [Lehn], it suffices to show that via the isomorphism $\Phi$ in (2.8), the linear operator $\mathfrak{g}_{k}$ on $\mathbb{Q}\left[q_{1}, q_{2}, \ldots\right]$ induced by the operator $\mathfrak{G}_{k}\left(1_{X}\right)$ on $\bigoplus_{n \geq 0} H^{*}\left(X^{[n]}\right)$ is given by

$$
\mathfrak{g}_{k}=\frac{(-1)^{k}}{(k+1) !} \sum_{n_{1}, \ldots, n_{k+1}>0} q_{n_{1}+\ldots+n_{k+1}} \partial_{n_{1}} \cdots \partial_{n_{k+1}},
$$

where $\partial_{i}=i \frac{\partial}{\partial q_{i}}$. Indeed, let $A=\mathfrak{a}_{-n_{1}}\left(1_{X}\right) \cdots \mathfrak{a}_{-n_{b}}\left(1_{X}\right)|0\rangle \in H^{*}\left(X^{[n]}\right)$, where $n_{1}, \ldots, n_{b}>0$ with $\sum_{\ell} n_{\ell}=n$. By Lemma 2.2. $\mathfrak{G}_{k}\left(1_{X}\right)(A)$ is equal to

$$
\sum_{i=0}^{k+1} \sum_{\sigma_{i}}\left(\prod_{\ell \in \sigma_{i}^{0}} \mathfrak{a}_{-n_{\ell}}\left(1_{X}\right)\right)\left[\left[\cdots\left[\mathfrak{G}_{k}\left(1_{X}\right), \mathfrak{a}_{-n_{\sigma_{i}(1)}}\left(1_{X}\right)\right], \cdots\right], \mathfrak{a}_{-n_{\sigma_{i}(i)}}\left(1_{X}\right)\right]|0\rangle
$$

where for each fixed $i, \sigma_{i}$ runs over all the maps $\{1, \ldots, i\} \rightarrow\{1, \ldots, b\}$ satisfying $\sigma_{i}(1)<\cdots<\sigma_{i}(i)$, and $\sigma_{i}^{0}=\left\{\ell \mid 1 \leq \ell \leq b, \ell \neq \sigma_{i}(1), \ldots, \sigma_{i}(i)\right\}$. Note from Lemma 2.1 that $\left[\left[\cdots\left[\mathfrak{a}_{t_{1}} \cdots \mathfrak{a}_{t_{r}}\left(\tau_{r *} \alpha\right), \mathfrak{a}_{-n_{\sigma_{i}(1)}}\left(1_{X}\right)\right], \cdots\right], \mathfrak{a}_{-n_{\sigma_{i}(i)}}\left(1_{X}\right)\right]|0\rangle \in \mathcal{I}^{[n]}$ if $i \leq r-2$ or $\alpha \in \mathcal{I}$. Hence by (2.11) and Theorem 2.4, $\mathfrak{G}_{k}\left(1_{X}\right)(A)$ equals

$$
\begin{aligned}
& \sum_{\sigma_{k+1}}\left(\prod_{\ell \in \sigma_{k+1}^{0}} \mathfrak{a}_{-n_{\ell}}\left(1_{X}\right)\right) \\
& \cdot\left[\left[\cdots\left[-\sum_{\ell(\lambda)=k+2,|\lambda|=0} \frac{1}{\lambda^{!}} \mathfrak{a}_{\lambda}\left(\tau_{*} 1_{X}\right), \mathfrak{a}_{-n_{\sigma_{k+1}(1)}}\left(1_{X}\right)\right], \cdots\right], \mathfrak{a}_{-n_{\sigma_{k+1}(k+1)}}\left(1_{X}\right)\right]|0\rangle
\end{aligned}
$$

modulo $\mathcal{I}^{[n]}$. By Lemma 2.1(i) again, the preceding expression is equal to

$$
\sum_{\sigma_{k+1}}\left(-\prod_{\ell=1}^{k+1}\left(-n_{\sigma_{k+1}(\ell)}\right)\right)\left(\prod_{\ell \in \sigma_{k+1}^{0}} \mathfrak{a}_{-n_{\ell}}\left(1_{X}\right)\right) \mathfrak{a}_{-n_{\sigma_{k+1}(1)}-\ldots-n_{\sigma_{k+1}(k+1)}}\left(1_{X}\right)|0\rangle .
$$

Therefore, we conclude that for the induced operator $\mathfrak{g}_{k}$,

$$
\begin{aligned}
\mathfrak{g}_{k}\left(q_{n_{1}} \cdots q_{n_{b}}\right) & =(-1)^{k} \sum_{\sigma_{k+1}}\left(\prod_{\ell=1}^{k+1} n_{\sigma_{k+1}(\ell)}\right)\left(\prod_{\ell \in \sigma_{k+1}^{0}} q_{n_{\ell}}\right) q_{n_{\sigma_{k+1}(1)}+\ldots+n_{\sigma_{k+1}(k+1)}} \\
& =\frac{(-1)^{k}}{(k+1) !} \sum_{\tilde{\sigma}_{k+1}}\left(\prod_{\ell=1}^{k+1} n_{\tilde{\sigma}_{k+1}(\ell)}\right)\left(\prod_{\ell \in \tilde{\sigma}_{k+1}^{0}} q_{n_{\ell}}\right) q_{n_{\tilde{\sigma}_{k+1}(1)}+\ldots+n_{\tilde{\sigma}_{k+1}(k+1)}},
\end{aligned}
$$

where $\tilde{\sigma}_{k+1}$ runs over all injective maps $\{1, \ldots, k+1\} \rightarrow\{1, \ldots, b\}$ and $\tilde{\sigma}_{k+1}^{0}=$ $\left\{\ell \mid 1 \leq \ell \leq b, \ell \neq \tilde{\sigma}_{k+1}(1), \ldots, \tilde{\sigma}_{k+1}(k+1)\right\}$ (so the only difference between $\tilde{\sigma}_{k+1}$ and $\sigma_{k+1}$ is that we have dropped the condition $\sigma_{k+1}(1)<\cdots<\sigma_{k+1}(k+1)$ ). Finally, the above formula for $\mathfrak{g}_{k}\left(q_{n_{1}} \cdots q_{n_{b}}\right)$ is equivalent to (2.10). 


\section{Partial $n$-Independence of structure constants FOR $X$ PROJECTIVE}

Given a finite set $S$ which is a disjoint union of subsets $S_{0}$ and $S_{1}$, we denote by $\mathcal{P}(S)$ the set of partition-valued functions $\rho=(\rho(c))_{c \in S}$ on $S$ such that for every $c \in S_{1}$, the partition $\rho(c)$ is required to be strict in the sense that $\rho(c)=$ $\left(1^{m_{1}(\rho(c))} 2^{m_{2}(\rho(c))} \ldots\right)$ with $m_{r}(\rho(c))=0$ or 1 for all $r \geq 1$.

Now let us take a linear basis $S=S_{0} \cup S_{1}$ of $H^{*}(X)$ such that $1_{X},[x] \in S_{0}, S_{0} \subset$ $H^{\text {even }}(X)$ and $S_{1} \subset H^{\text {odd }}(X)$. If we write $\rho=(\rho(c))_{c \in S}$ and $\rho(c)=\left(r^{m_{r}(\rho(c))}\right)_{r \geq 1}=$ $\left(1^{m_{1}(\rho(c))} 2^{m_{2}(\rho(c))} \ldots\right)$, then we put $\ell(\rho)=\sum_{c \in S} \ell(\rho(c))=\sum_{c \in S, r \geq 1} m_{r}(\rho(c))$ and

$$
\|\rho\|=\sum_{c \in S}|\rho(c)|=\sum_{c \in S, r \geq 1} r \cdot m_{r}(\rho(c)) .
$$

Given $\rho \in \mathcal{P}(S)$ and $n \geq\|\rho\|+\ell\left(\rho\left(1_{X}\right)\right)$, we define $\tilde{\rho} \in \mathcal{P}(S)$ by putting $m_{r}(\tilde{\rho}(c))=$ $m_{r}(\rho(c))$ for $c \in S-\left\{1_{X}\right\}, m_{1}\left(\tilde{\rho}\left(1_{X}\right)\right)=n-\|\rho\|-\ell\left(\rho\left(1_{X}\right)\right)$, and $m_{r}\left(\tilde{\rho}\left(1_{X}\right)\right)=$ $m_{r-1}\left(\rho\left(1_{X}\right)\right)$ for $r \geq 2$. Note that $\|\tilde{\rho}\|=n$. We define $\mathfrak{b}_{\rho}(n) \in H^{*}\left(X^{[n]}\right)$ by

$$
\begin{aligned}
\mathfrak{b}_{\rho}(n) & =\frac{1}{\prod_{r \geq 1}\left(r^{m_{r}\left(\tilde{\rho}\left(1_{X}\right)\right)} m_{r}\left(\tilde{\rho}\left(1_{X}\right)\right) !\right)}\left(\prod_{c \in S} \prod_{r \geq 1} \mathfrak{a}_{-r}(c)^{m_{r}(\tilde{\rho}(c))}\right)|0\rangle \\
& =\frac{\mathbf{1}_{-\left(n-\|\rho\|-\ell\left(\rho\left(1_{X}\right)\right)\right)}}{\prod_{r \geq 2}\left(r^{m_{r}\left(\tilde{\rho}\left(1_{X}\right)\right)} m_{r}\left(\tilde{\rho}\left(1_{X}\right)\right) !\right)}\left(\prod_{\substack{c \in S, r \geq 1 \\
c \neq 1 X \text { or } r>1}} \mathfrak{a}_{-r}(c)^{m_{r}(\tilde{\rho}(c))}\right)|0\rangle,
\end{aligned}
$$

where we fix the order of the elements $c \in S_{1}$ appearing in the product $\prod_{c \in S}$ once and for all. For $0 \leq n<\|\rho\|+\ell\left(\rho\left(1_{X}\right)\right)$, we set $\mathfrak{b}_{\rho}(n)=0$. This is consistent with (3.2) and Definition 2.3(iii). We remark that the only part in $\mathfrak{b}_{\rho}(n)$ involving $n$ is

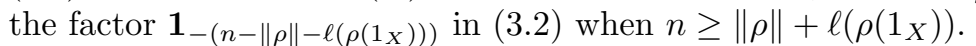

As a corollary to the theorem of Nakajima and Grojnowski [Gro], [Na1], Na2], $H^{*}\left(X^{[n]}\right)$ has a linear basis consisting of the classes

$$
\mathfrak{b}_{\rho}(n), \quad \rho \in \mathcal{P}(S) \text { and }\|\rho\|+\ell\left(\rho\left(1_{X}\right)\right) \leq n .
$$

Fix a positive integer $n$ and $\rho, \sigma \in \mathcal{P}(S)$ satisfying $\|\rho\|+\ell\left(\rho\left(1_{X}\right)\right) \leq n$ and $\|\sigma\|+$ $\ell\left(\sigma\left(1_{X}\right)\right) \leq n$. Then we can write the cup product $\mathfrak{b}_{\rho}(n) \cdot \mathfrak{b}_{\sigma}(n)$ as

$$
\mathfrak{b}_{\rho}(n) \cdot \mathfrak{b}_{\sigma}(n)=\sum_{\nu \in \mathcal{P}(S)} a_{\rho \sigma}^{\nu}(n) \mathfrak{b}_{\nu}(n),
$$

where we have used $a_{\rho \sigma}^{\nu}(n) \in \mathbb{Q}$ to denote the structure constants.

Proposition 3.1. Let $X$ be a smooth projective surface. The structure constants $a_{\rho \sigma}^{\nu}(n)$ of the cohomology ring $H^{*}\left(X^{[n]}\right)$ are polynomials in $n$ of degree at most

$$
\left(\|\rho\|+\ell\left(\rho\left(1_{X}\right)\right)\right)+\left(\|\sigma\|+\ell\left(\sigma\left(1_{X}\right)\right)\right)-\left(\|\nu\|+\ell\left(\nu\left(1_{X}\right)\right)\right) .
$$

Proof. This is a consequence of the much more powerful Theorem 5.1 in [LQW3. More explicitly, let $f(\rho)=\prod_{r>2}\left(r^{m_{r}\left(\tilde{\rho}\left(1_{X}\right)\right)} m_{r}\left(\tilde{\rho}\left(1_{X}\right)\right) !\right)$. Then, $f(\rho)$ is independent of $n$. By Theorem 5.1 in LQW3, the cup product $f(\rho) \mathfrak{b}_{\rho}(n) \cdot f(\sigma) \mathfrak{b}_{\sigma}(n)$ is a linear combination of expressions of the form

$$
\frac{\left(n-\|\nu\|-\ell\left(\nu\left(1_{X}\right)\right)\right) !}{\left(n-\|\nu\|-\ell\left(\nu\left(1_{X}\right)\right)-i\right) !} \cdot f(\nu) \mathfrak{b}_{\nu}(n),
$$


such that $i \geq 0,\left(\|\nu\|+\ell\left(\nu\left(1_{X}\right)\right)+i\right) \leq\left(\|\rho\|+\ell\left(\rho\left(1_{X}\right)\right)\right)+\left(\|\sigma\|+\ell\left(\sigma\left(1_{X}\right)\right)\right)$, and all the coefficients in this linear combination are independent of $n$. It follows that all the structure constants $a_{\rho \sigma}^{\nu}(n)$ are polynomials in $n$ of degree at most (3.5).

To state our main result in this section, let $\mathcal{I}=H^{4}(X)$ and $S_{\mathcal{I}}=\{[x]\} \subset S$. Regard $\mathcal{P}\left(S-S_{\mathcal{I}}\right) \subset \mathcal{P}(S)$. Then, 3.4 implies that

$$
\mathfrak{b}_{\rho}(n) \cdot \mathfrak{b}_{\sigma}(n) \equiv \sum_{\nu \in \mathcal{P}\left(S-S_{\mathcal{I}}\right)} a_{\rho \sigma}^{\nu}(n) \mathfrak{b}_{\nu}(n) \quad\left(\bmod \mathcal{I}^{[n]}\right)
$$

Theorem 3.2. Let $X$ be a smooth projective surface, and $\mathcal{I}=H^{4}(X)$. Then, all the structure constants $a_{\rho \sigma}^{\nu}(n)$ in (3.6) are independent of $n$.

To prove this theorem, we first need to establish four technical lemmas.

Lemma 3.3. If $k \geq 2$ and $\alpha$ is homogeneous, then $\tau_{k *} \alpha=\sum_{j} \alpha_{j, 1} \otimes \cdots \otimes \alpha_{j, k}$, where for each $j$, either $\left|\alpha_{j, \ell}\right|=4$ for some $\ell$, or $0<\left|\alpha_{j, \ell}\right|<4$ for every $\ell$.

Proof. Assume $\left|\alpha_{j, \ell}\right|<4$ for every $\ell$. If $\left|\alpha_{j, \ell}\right|=0$ for some $\ell$, then $4(k-1)+|\alpha|=$ $\left|\tau_{k *} \alpha\right|=\sum_{i=1}^{k}\left|\alpha_{j, i}\right| \leq 3(k-1)$. So $(k-1)+|\alpha| \leq 0$, contradicting $k \geq 2$.

Lemma 3.4. Let $\mathcal{I}=H^{4}(X), s \geq 0, n_{1}, \ldots, n_{s}>0, \tilde{n}=\sum_{\ell=1}^{s} n_{\ell}$, and $n \geq \tilde{n}$. Let $\alpha, \alpha_{1}, \ldots, \alpha_{s} \in H^{*}(X)$ be homogeneous. Assume $k+|\alpha| \geq 1$ and $n_{\ell}+\left|\alpha_{\ell}\right| \geq 2$ for every $\ell$. Then modulo $\mathcal{I}^{[n]}$, the cup product

$$
G_{k}(\alpha, n) \cdot\left(\mathbf{1}_{-(n-\tilde{n})} \mathfrak{a}_{-n_{1}}\left(\alpha_{1}\right) \cdots \mathfrak{a}_{-n_{s}}\left(\alpha_{s}\right)|0\rangle\right)
$$

is a universal linear combination of the basis (3.3).

Proof. By Lemma 2.7)(i) and (ii), the statement is trivial if one of the classes $\alpha, \alpha_{1}, \ldots, \alpha_{s} \in H^{*}(X)$ is contained in $\mathcal{I}$. So in the rest of the proof, we assume that none of the classes $\alpha, \alpha_{1}, \ldots, \alpha_{s}$ is contained in $\mathcal{I}$.

Our argument is similar to the proof of Lemma 2.7 $(\mathrm{i})$. Put $\mathfrak{g}=\mathfrak{G}_{k}(\alpha)$. Then, $B \stackrel{\text { def }}{=} G_{k}(\alpha, n) \cdot \mathbf{1}_{-(n-\tilde{n})} \mathfrak{a}_{-n_{1}}\left(\alpha_{1}\right) \cdots \mathfrak{a}_{-n_{s}}\left(\alpha_{s}\right)|0\rangle$ is equal to

$$
\frac{1}{(n-\tilde{n}) !} \mathfrak{g a} \mathfrak{a}_{-1}\left(1_{X}\right)^{n-\tilde{n}} \mathfrak{a}_{-n_{1}}\left(\alpha_{1}\right) \cdots \mathfrak{a}_{-n_{s}}\left(\alpha_{s}\right)|0\rangle
$$

By Lemma 2.2 $B$ is a universal finite linear combination of expressions of the form

$$
\begin{aligned}
& \frac{1}{(n-\tilde{n}) !}\left(\begin{array}{c}
n-\tilde{n} \\
j
\end{array}\right) \mathfrak{a}_{-1}\left(1_{X}\right)^{n-\tilde{n}-j}\left(\prod_{\ell \in \sigma_{i}^{0}} \mathfrak{a}_{-n_{\ell}}\left(\alpha_{\ell}\right)\right) \\
& \cdot\left[\left[\cdots \left[\left[\left[[\mathfrak{g}, \underbrace{\left.\left.\left.\mathfrak{a}_{-1}\left(1_{X}\right)\right], \ldots\right], \mathfrak{a}_{-1}\left(1_{X}\right)\right]}_{j \text { times }}, \mathfrak{a}_{-n_{\sigma_{i}(1)}}\left(\alpha_{\sigma_{i}(1)}\right)], \cdots\right], \mathfrak{a}_{-n_{\sigma_{i}(i)}}\left(\alpha_{\sigma_{i}(i)}\right)\right]|0\rangle,\right.\right.\right.
\end{aligned}
$$

where $0 \leq j \leq n-\tilde{n}, 0 \leq i \leq s, 0 \leq j+i \leq k+1, \sigma_{i}$ maps the set $\{1, \ldots, i\}$ to the set $\{1, \ldots, s\}$ with $\sigma_{i}(1)<\cdots<\sigma_{i}(i)$, and $\sigma_{i}^{0}=\left\{\ell \mid 1 \leq \ell \leq s, \ell \neq \sigma_{i}(1), \ldots, \sigma_{i}(i)\right\}$. By Theorem 2.4 and Lemma 2.1(i), we conclude that

$$
\left[\left[\cdots \left[\left[\left[[\mathfrak{g}, \underbrace{\left.\left.\left.\mathfrak{a}_{-1}\left(1_{X}\right)\right], \ldots\right], \mathfrak{a}_{-1}\left(1_{X}\right)\right]}_{j \text { times }}, \mathfrak{a}_{-n_{\sigma_{i}(1)}}\left(\alpha_{\sigma_{i}(1)}\right)], \cdots\right], \mathfrak{a}_{-n_{\sigma_{i}(i)}}\left(\alpha_{\sigma_{i}(i)}\right)\right]|0\rangle\right.\right.\right.
$$

is a universal finite linear combination of expressions $\mathfrak{a}_{-\lambda}\left(\tau_{*}\left(\epsilon \alpha \alpha_{\sigma_{i}(1)} \cdots \alpha_{\sigma_{i}(i)}\right)\right)|0\rangle$, where $\epsilon \in\left\{1_{X}, e, K, K^{2}\right\}, \lambda \vdash j+n_{\sigma_{i}(1)}+\cdots+n_{\sigma_{i}(i)}$ and $\ell(\lambda)=k+2-|\epsilon| / 2-(j+i)$. 
So $B$ is a universal finite linear combination of expressions

$$
\mathbf{1}_{-(n-\tilde{n}-j)}\left(\prod_{\ell \in \sigma_{i}^{0}} \mathfrak{a}_{-n_{\ell}}\left(\alpha_{\ell}\right)\right) \mathfrak{a}_{-\lambda}\left(\tau_{*}\left(\epsilon \alpha \alpha_{\sigma_{i}(1)} \cdots \alpha_{\sigma_{i}(i)}\right)\right)|0\rangle,
$$

where $\lambda \vdash j+n_{\sigma_{i}(1)}+\cdots+n_{\sigma_{i}(i)}$ and $\ell(\lambda)=k+2-|\epsilon| / 2-(j+i)$.

To prove our lemma, we see from (3.2) that it suffices to show that modulo $\mathcal{I}^{[\tilde{n}+j]}$, the part $\left(\prod_{\ell \in \sigma_{i}^{0}} \mathfrak{a}_{-n_{\ell}}\left(\alpha_{\ell}\right)\right) \mathfrak{a}_{-\lambda}\left(\tau_{*}\left(\epsilon \alpha \alpha_{\sigma_{i}(1)} \cdots \alpha_{\sigma_{i}(i)}\right)\right)|0\rangle$ in (3.8) does not contain $\mathfrak{a}_{-1}\left(1_{X}\right)$. Since $n_{\ell}+\left|\alpha_{\ell}\right| \geq 2$ for every $\ell$, this is equivalent to show that modulo $\mathcal{I}^{[\ell(\lambda)]}$, the part $\mathfrak{a}_{-\lambda}\left(\tau_{*}\left(\epsilon \alpha \alpha_{\sigma_{i}(1)} \cdots \alpha_{\sigma_{i}(i)}\right)\right)|0\rangle$ in (3.8) does not contain $\mathfrak{a}_{-1}\left(1_{X}\right)$. By Lemma 3.3 this is true if $\ell(\lambda) \geq 2$. So let $\ell(\lambda)=1$. Then, we have

$$
\mathfrak{a}_{-\lambda}\left(\tau_{*}\left(\epsilon \alpha \alpha_{\sigma_{i}(1)} \cdots \alpha_{\sigma_{i}(i)}\right)\right)|0\rangle=\mathfrak{a}_{-t}\left(\epsilon \alpha \alpha_{\sigma_{i}(1)} \cdots \alpha_{\sigma_{i}(i)}\right)|0\rangle
$$

where $t=|\lambda|=j+n_{\sigma_{i}(1)}+\cdots+n_{\sigma_{i}(i)}$ and $k+2-|\epsilon| / 2-(j+i)=1$.

If $\mathfrak{a}_{-\lambda}\left(\tau_{*}\left(\epsilon \alpha \alpha_{\sigma_{i}(1)} \cdots \alpha_{\sigma_{i}(i)}\right)\right)|0\rangle$ contains $\mathfrak{a}_{-1}\left(1_{X}\right)$, then we must have $t=1$ and $\left|\epsilon \alpha \alpha_{\sigma_{i}(1)} \cdots \alpha_{\sigma_{i}(i)}\right|=0$. So $j+n_{\sigma_{i}(1)}+\cdots+n_{\sigma_{i}(i)}=1, \epsilon=1_{X}$, and $|\alpha|=\left|\alpha_{\sigma_{i}(1)}\right|=$ $\cdots=\left|\alpha_{\sigma_{i}(i)}\right|=0$. Thus, either $j=0, i=1, n_{\sigma_{1}(1)}=1$ and $\left|\alpha_{\sigma_{1}(1)}\right|=0$, or $j=1$ and $i=0$. The first case contradicts to $n_{\sigma_{1}(1)}+\left|\alpha_{\sigma_{1}(1)}\right| \geq 2$. In the second case, we see from $k+2-|\epsilon| / 2-(j+i)=1$ that $k=0$, contradicting $k+|\alpha| \geq 1$. So $\mathfrak{a}_{-\lambda}\left(\tau_{*}\left(\epsilon \alpha \alpha_{\sigma_{i}(1)} \cdots \alpha_{\sigma_{i}(i)}\right)\right)|0\rangle$ cannot contain $\mathfrak{a}_{-1}\left(1_{X}\right)$.

Lemma 3.5. Let $\mathcal{I}=H^{4}(X), s \geq 1, k_{1}, \ldots, k_{s} \geq 0, k_{i}+\left|\alpha_{i}\right| \geq 1$ for every $i$. Then

(i) modulo $\mathcal{I}^{[n]}, \prod_{i=1}^{s} G_{k_{i}}\left(\alpha_{i}, n\right)$ is a universal linear combination of (3.3), and

(ii) when $n \geq n_{0} \stackrel{\text { def }}{=} \sum_{i=1}^{s}\left(k_{i}+1\right)$, the leading term in the cup product $\prod_{i=1}^{s} G_{k_{i}}\left(\alpha_{i}, n\right)$ is equal to $\left(\prod_{i=1}^{s} \frac{(-1)^{k_{i}}}{\left(k_{i}+1\right) !}\right) \mathbf{1}_{-\left(n-n_{0}\right)}\left(\prod_{i=1}^{s} \mathfrak{a}_{-\left(k_{i}+1\right)}\left(\alpha_{i}\right)\right)|0\rangle$, which is equal to a universal multiple of $\mathfrak{b}_{\nu}(n)$ for some $\nu \in \mathcal{P}(S)$.

Proof. (i) Use induction on $s$. When $s=1$, our statement follows from Lemma 3.4 (take the integer $s$ there to be 0). Now, assume that the statement is true for $s-1$ with $s \geq 2$, i.e., $\prod_{i=1}^{s-1} G_{k_{i}}\left(\alpha_{i}, n\right)$ is a universal finite linear combination of the basis classes $\mathfrak{b}_{\nu}(n), \nu \in \mathcal{P}(S)$ and $\|\nu\|+\ell\left(\rho\left(1_{X}\right)\right) \leq n$. Note from (3.2) that up to some universal factor, every basis class $\mathfrak{b}_{\nu}(n)$ is of the form $\mathbf{1}_{-(n-\tilde{n})} \mathfrak{a}_{-n_{1}}\left(\beta_{1}\right) \ldots$ $\mathfrak{a}_{-n_{s}}\left(\beta_{s}\right)|0\rangle$, where $n_{1}, \ldots, n_{s}>0, \tilde{n}=n_{1}+\ldots+n_{s}, n \geq \tilde{n}$, and $n_{\ell}+\left|\beta_{\ell}\right| \geq 2$ for every $\ell$. So by Lemma 3.4, our statement for $s$ follows.

(ii) The statement about the leading term comes from the proof of Theorem 2.9 while the other statement about the universal multiple follows from (3.2). 
Lemma 3.6. Let $\mathcal{I}=H^{4}(X)$. Then modulo $\mathcal{I}^{[n]}$, the basis element $\mathfrak{b}_{\rho}(n)$ is a universal finite linear combination of products of the form $\prod_{j=1}^{t} G_{m_{j}}\left(\beta_{j}, n\right)$, where $m_{j}+\left|\beta_{j}\right| \geq 1$ for each $j$, and $\sum_{j=1}^{t}\left(m_{j}+1\right) \leq\|\rho\|+\ell\left(\rho\left(1_{X}\right)\right)$.

Proof. By (3.2), up to some universal factor, the basis class $\mathfrak{b}_{\rho}(n)$ is of the form $\mathbf{1}_{-\left(n-n_{0}\right)}\left(\prod_{i=1}^{s} \mathfrak{a}_{-\left(k_{i}+1\right)}\left(\alpha_{i}\right)\right)|0\rangle$, where $n \geq n_{0} \stackrel{\text { def }}{=}\|\rho\|+\ell\left(\rho\left(1_{X}\right)\right)=\sum_{i=1}^{s}\left(k_{i}+1\right)$, and $k_{i} \geq 0$ and $k_{i}+\left|\alpha_{i}\right| \geq 1$ for every $i$. Now our result follows from an induction on $n_{0}$ the same way as in the proof of Theorem 2.9, and Lemma 3.5(i) and (ii).

Proof of Theorem 3.2. By Lemma 3.6, $\mathfrak{b}_{\rho}(n)$ is a universal finite linear combination of expressions of the form $\prod_{j=1}^{t_{1}} G_{m_{1, j}}\left(\beta_{1, j}, n\right)$, where $m_{1, j}+\left|\beta_{1, j}\right| \geq 1$ for every $j$. Similarly, $\mathfrak{b}_{\sigma}(n)$ is a universal finite linear combination of expressions of the form $\prod_{j=1}^{t_{2}} G_{m_{2, j}}\left(\beta_{2, j}, n\right)$, where $m_{1, j}+\left|\beta_{1, j}\right| \geq 1$ for every $j$. Therefore, $\mathfrak{b}_{\rho}(n) \cdot \mathfrak{b}_{\sigma}(n)$ is a universal finite linear combination of expressions of the form $\prod_{j=1}^{t} G_{m_{j}}\left(\beta_{j}, n\right)$, where $m_{j}+\left|\beta_{j}\right| \geq 1$ for every $j$. Now our result follows from Lemma $3.5(\mathrm{i})$.

We end this section with a lemma to be used in the next section. For convenience, when $\ell(\rho)=1$, i.e., when the partition $\rho(c)$ is a one-part partition $(r)$ for some $c \in S$ and is empty for all the other elements in $S$, we will simply write $\mathfrak{b}_{\rho}(n)=\mathfrak{b}_{r, c}(n)$.

Lemma 3.7. Let $\mathcal{I}=H^{4}(X)$. Then modulo $\mathcal{I}^{[n]}$, the basis class $\mathfrak{b}_{\rho}(n)$ is a universal finite linear combination of products of the form $\prod_{j=1}^{t} \mathfrak{b}_{r_{j}, c_{j}}(n)$.

Proof. Note from (3.2) that $\mathfrak{b}_{r, c}(n)=\mathbf{1}_{-(n-r)} \mathfrak{a}_{-r}(c)|0\rangle$ if $c \neq 1_{X}$, while $\mathfrak{b}_{r, c}(n)=$ $\mathbf{1}_{-(n-r-1)} \mathfrak{a}_{-(r+1)}(c)|0\rangle$ if $c=1_{X}$. So by Lemma 3.6, it suffices to show that if $c \in S$ and $k+|c| \geq 1$, then modulo $\mathcal{I}^{[n]}, G_{k}(c, n)$ is a universal finite linear combination of products of the form $\prod_{j=1}^{t}\left(\mathbf{1}_{-\left(n-r_{j}\right)} \mathfrak{a}_{-r_{j}}\left(c_{j}\right)|0\rangle\right)$, where $r_{j} \geq 1, c_{j} \in S$ and $r_{j}+\left|c_{j}\right| \geq 2$.

Use induction on $k$. When $k=0$, we have $G_{0}(c, n)=\mathbf{1}_{-(n-1)} \mathfrak{a}_{-1}(c)|0\rangle$ by formula (2.6). Next, we prove that the statement in the preceding paragraph holds for $k \geq 1$ by assuming that it is true for $0, \ldots, k-1$. By Lemma 3.5 (i) and (ii), modulo $\mathcal{I}^{[n]}, G_{k}(c, n)-(-1)^{k} /(k+1) ! \cdot \mathbf{1}_{-(n-k-1)} \mathfrak{a}_{-(k+1)}(c)|0\rangle$ is a universal linear combination of the basis elements $\mathfrak{b}_{\rho}(n)$ satisfying $\|\rho\|+\ell\left(\rho\left(1_{X}\right)\right)<(k+1)$. By Lemma 3.6, modulo $\mathcal{I}^{[n]}$, each $\mathfrak{b}_{\rho}(n)$ is a universal finite linear combination of products of the form $\prod_{j=1}^{t} G_{k_{j}}\left(\gamma_{j}, n\right)$, where $\gamma_{j} \in S, k_{j}+\left|\gamma_{j}\right| \geq 1$, and $\sum_{j=1}^{t}\left(k_{j}+1\right) \leq$ $\|\rho\|+\ell\left(\rho\left(1_{X}\right)\right)$. Note that $k_{j}<k$ for every $j$. So by induction, we conclude that 
modulo $\mathcal{I}^{[n]}, G_{k}(c, n)$ is a universal finite linear combination of products of the form $\prod_{j=1}^{t}\left(\mathbf{1}_{-\left(n-r_{j}\right)} \mathfrak{a}_{-r_{j}}\left(c_{j}\right)|0\rangle\right)$, where $r_{j} \geq 1, c_{j} \in S$ and $r_{j}+\left|c_{j}\right| \geq 2$.

\section{The cohomology Ring $H^{*}\left(X^{[n]}\right)$ For $X$ Quasi-Projective}

In this section, we will apply our results in previous sections to smooth quasiprojective surfaces. Our terminology "quasi-projective" means "quasi-projective but not projective". Recall from Na1 that for a smooth quasi-projective surface $X$, the creation operators are modelled on the Borel-Moore homology $H_{*}^{B M}(X)$, while the annihilation operators are modelled on the ordinary homology $H_{*}(X)$. Then the Fock space of the Heisenberg algebra is taken to be the direct sum over all $n$ of the Borel-Moore homology groups $H_{*}^{B M}\left(X^{[n]}\right)$ [Na1]. Let $H_{c}^{*}(X)$ be the cohomology with compact support. Using the the Poincaré dualities PD : $H^{4-i}(X) \rightarrow H_{i}^{B M}(X)$ and PD : $H_{c}^{4-i}(X) \rightarrow H_{i}(X)$, we can regard the creation operators $\mathfrak{a}_{-n}(\alpha)$ with $n>0$ as being modelled on $H^{*}(X)$ (i.e., $\alpha \in H^{*}(X)$ ), while we can regard the annihilation operators $\mathfrak{a}_{n}(\beta)$ with $n>0$ as being modelled on $H_{c}^{*}(X)$ (i.e., $\beta \in$ $\left.H_{c}^{*}(X)\right)$. Accordingly, with the help of the Poincaré duality PD : $H^{4 n-i}\left(X^{[n]}\right) \rightarrow$ $H_{i}^{B M}\left(X^{[n]}\right)$, from now on we can take the Fock space to be the direct sum of the ordinary cohomology groups $H^{*}\left(X^{[n]}\right)$.

4.1. The $n$-independence of the structure constants. Let $X$ be a smooth quasi-projective surface embedded in a smooth projective surface $\bar{X}$, and let $\iota$ : $X \rightarrow \bar{X}$ be the inclusion map. Then we have induced embeddings $\iota_{n}: X^{[n]} \rightarrow \bar{X}^{[n]}$ for $n \geq 0$, and induced ring homomorphisms $\iota_{n}^{*}: H^{*}\left(\bar{X}^{[n]}\right) \rightarrow H^{*}\left(X^{[n]}\right)$. The maps $\iota^{*}$ and $\iota_{n}^{*}$ are related by the following.

Lemma 4.1. Let notations be as in the preceding paragraph. Then,

$$
\iota_{n}^{*}\left(\mathfrak{a}_{-n_{1}}\left(\bar{\alpha}_{1}\right) \cdots \mathfrak{a}_{-n_{k}}\left(\bar{\alpha}_{k}\right)|0\rangle\right)=\mathfrak{a}_{-n_{1}}\left(\iota^{*} \bar{\alpha}_{1}\right) \cdots \mathfrak{a}_{-n_{k}}\left(\iota^{*} \bar{\alpha}_{k}\right)|0\rangle,
$$

where $k \geq 0, n_{1}, \ldots, n_{k}>0$, and $n_{1}+\ldots+n_{k}=n$.

Proof. For $n \geq 0$, let $\left(\iota_{n}^{*}\right)^{B M}: H_{i}^{B M}\left(\bar{X}^{[n]}\right) \rightarrow H_{i}^{B M}\left(X^{[n]}\right)$ be the natural map induced by the embedding $\iota_{n}: X^{[n]} \rightarrow \bar{X}^{[n]}$. Then, it is well known that $\left(\iota_{n}^{*}\right)^{B M}$ 。 $\mathrm{PD}=\mathrm{PD} \circ\left(\iota_{n}^{*}\right)^{B M}$ (see Na2]). Combining with Nakajima's constructions in [Na1, we obtain $\iota_{m_{1}+m_{2}}^{*} \mathfrak{a}_{-m_{2}}(\bar{\alpha})(A)=\mathfrak{a}_{-m_{2}}\left(\iota^{*} \bar{\alpha}\right) \iota_{m_{1}}^{*}(A)$ for $m_{1}>0, m_{2}>0$ and $A \in$ $H^{*}\left(\bar{X}^{\left[m_{1}\right]}\right)$. Applying this repeatedly, we obtain (4.1).

Next, assume that $\iota^{*}: H^{*}(\bar{X}) \rightarrow H^{*}(X)$ is surjective. Let $\mathcal{I}=\operatorname{ker}\left(\iota^{*}\right)$. Fix a linear basis $S$ of $H^{*}(\bar{X})$ as in Section 3 such that $S$ contains a linear basis $S_{\mathcal{I}}$ of $\mathcal{I}$ and $S_{X} \stackrel{\text { def }}{=} \iota^{*}\left(S-S_{\mathcal{I}}\right)$ is a linear basis of $H^{*}(X)$. By Lemma 4.1, $\operatorname{ker}\left(\iota_{n}^{*}\right)=\mathcal{I}^{[n]}$ which is defined in Definition [2.6. Also, a linear basis of $H^{*}\left(X^{[n]}\right)$ is given by

$$
\mathfrak{b}_{\rho_{X}}(n), \quad \rho_{X} \in \mathcal{P}\left(S_{X}\right) \text { and }\left\|\rho_{X}\right\|+\ell\left(\rho_{X}\left(1_{X}\right)\right) \leq n,
$$

where $\mathfrak{b}_{\rho_{X}}(n)$ is defined in a similar way as in (3.1) and (3.2). So for $\rho_{X}, \sigma_{X} \in$ $\mathcal{P}\left(S_{X}\right)$, we can write the cup product $\mathfrak{b}_{\rho_{X}}(n) \cdot \mathfrak{b}_{\sigma_{X}}(n)$ as

$$
\mathfrak{b}_{\rho_{X}}(n) \cdot \mathfrak{b}_{\sigma_{X}}(n)=\sum_{\nu_{X} \in \mathcal{P}\left(S_{X}\right)} a_{\rho_{X} \sigma_{X}}^{\nu_{X}}(n) \mathfrak{b}_{\nu_{X}}(n)
$$

where $a_{\rho_{X} \sigma_{X}}^{\nu_{X}}(n) \in \mathbb{Q}$ stands for the structure constants. 
Definition 4.2. A smooth quasi-projective surface $X$ satisfies the $S$-property if it can be embedded in a smooth projective surface $\bar{X}$ such that the induced ring homomorphism $H^{*}(\bar{X}) \rightarrow H^{*}(X)$ is surjective.

Theorem 4.3. Let $X$ be a smooth quasi-projective surface satisfying the S-property. Then all the structure constants $a_{\rho_{X} \sigma_{X}}^{\nu_{X}}(n)$ in (4.3) are independent of $n$.

Proof. Let notations be as above. Note that $\iota^{*}:\left(S-S_{\mathcal{I}}\right) \rightarrow S_{X}$ is bijective. Define $\rho, \sigma \in \mathcal{P}(S)$ by putting $m_{r}(\rho(\bar{c}))=m_{r}\left(\rho_{X}\left(\iota^{*} \bar{c}\right)\right)$ and $m_{r}(\sigma(\bar{c}))=m_{r}\left(\sigma_{X}\left(\iota^{*} \bar{c}\right)\right)$ when $\bar{c} \in\left(S-S_{\mathcal{I}}\right)$, and $m_{r}(\rho(\bar{c}))=0=m_{r}(\sigma(\bar{c}))$ when $\bar{c} \in S_{\mathcal{I}}$. By Theorem 3.2,

$$
\mathfrak{b}_{\rho}(n) \cdot \mathfrak{b}_{\sigma}(n) \equiv \sum_{\nu \in \mathcal{P}(S-\{[x]\})} a_{\rho \sigma}^{\nu}(n) \mathfrak{b}_{\nu}(n) \quad\left(\bmod \overline{\mathcal{I}}^{[n]}\right)
$$

where $\overline{\mathcal{I}}=H^{4}(\bar{X})$ and all the $a_{\rho \sigma}^{\nu}(n)$ are independent of $n$. Since $\overline{\mathcal{I}} \subset \mathcal{I}$,

$$
\mathfrak{b}_{\rho}(n) \cdot \mathfrak{b}_{\sigma}(n) \equiv \sum_{\nu \in \mathcal{P}\left(S-S_{\mathcal{I}}\right)} a_{\rho \sigma}^{\nu}(n) \mathfrak{b}_{\nu}(n) \quad\left(\bmod \mathcal{I}^{[n]}\right),
$$

where all the structure constants $a_{\rho \sigma}^{\nu}(n)$ are independent of $n$. By Lemma 4.1 $\iota_{n}^{*}\left(\mathfrak{b}_{\rho}(n)\right)=\mathfrak{b}_{\rho_{X}}(n)$ and $\iota_{n}^{*}\left(\mathfrak{b}_{\sigma}(n)\right)=\mathfrak{b}_{\sigma_{X}}(n)$. Therefore, applying $\iota_{n}^{*}$ to (4.4), we see that all the structure constants $a_{\rho_{X} \sigma_{X}}^{\nu_{X}}(n)$ in (4.3) are independent of $n$.

Thanks to Theorem 4.3 we will simply denote the structure constants $a_{\rho_{X}}^{\nu_{X}} \sigma_{X}(n)$ in (4.3) by $a_{\rho_{X} \sigma_{X}}^{\nu_{X}}$. Next, we study ring generators for the cohomology ring $H^{*}\left(X^{[n]}\right)$ when $X$ is a smooth quasi-projective surface satisfying the S-property. For $\alpha \in$ $H^{*}(X)$, define $G_{k}(\alpha, n)=\iota_{n}^{*} G_{k}(\bar{\alpha}, n)$, where $\bar{\alpha} \in H^{*}(\bar{X})$ satisfies $\iota^{*} \bar{\alpha}=\alpha$. This is independent of the choice of $\bar{\alpha}$ by Theorem 2.9 and the linearity of $G_{k}(\bar{\alpha}, n)$ in $\bar{\alpha}$.

Theorem 4.4. Let $X$ be a smooth quasi-projective surface embedded in a smooth projective surface $\bar{X}$ such that the induced map $H^{*}(\bar{X}) \rightarrow H^{*}(X)$ is surjective. Then, the cohomology classes $G_{k}(\alpha, n)$, as $0 \leq k<n$ and $\alpha$ runs over a linear basis of $H^{*}(X)$ form a set of ring generators of $H^{*}\left(X^{[n]}\right)$.

Proof. Let notations be as above. By Lemma 4.1 $H^{*}\left(X^{[n]}\right) \cong H^{*}\left(\bar{X}^{[n]}\right) / \mathcal{I}^{[n]}$. Recall that the classes $G_{k}(\bar{\alpha}, n), 0 \leq k<n$ and $\bar{\alpha} \in S \subset H^{*}(\bar{X})$ form a set of ring generators of $H^{*}\left(\bar{X}^{[n]}\right)$. It follows from Theorem 2.9 that the classes $\iota_{n}^{*} G_{k}(\bar{\alpha}, n)$, $0 \leq k<n$ and $\bar{\alpha} \in\left(S-S_{\mathcal{I}}\right)$ form a set of ring generators of $H^{*}\left(X^{[n]}\right)$. In other words, the classes $G_{k}(\alpha, n), 0 \leq k<n$ and $\alpha \in S_{X}=\iota^{*}\left(S-S_{\mathcal{I}}\right)$ form a set of ring generators of $H^{*}\left(X^{[n]}\right)$. Finally, note that $S_{X}$ is a linear basis of $H^{*}(X)$.

4.2. A universal ring. Let $\mathfrak{A}=-\mathfrak{a}_{1}\left([x]_{c}\right)$, where $[x]_{c} \in H_{c}^{4}(X)$ is the Poincaré dual of the homology class in $H_{0}(X)$ represented by a point in $X$.

Lemma 4.5. Let $X$ be a smooth quasi-projective surface. Then the linear map $\mathfrak{A}: H^{*}\left(X^{[n+1]}\right) \rightarrow H^{*}\left(X^{[n]}\right)$ is surjective. In fact, it sends $\mathfrak{b}_{\rho_{X}}(n+1)$ to $\mathfrak{b}_{\rho_{X}}(n)$.

Proof. Follows from the definition of the cohomology classes $\mathfrak{b}_{\rho_{X}}(n)$.

Corollary 4.6. Let $X$ be a smooth quasi-projective surface which satisfies the $S$ property. Then $\mathfrak{A}: H^{*}\left(X^{[n+1]}\right) \rightarrow H^{*}\left(X^{[n]}\right)$ is a surjective ring homomorphism.

Proof. Follows immediately from Lemma 4.5 and Theorem 4.3. 
Remark 4.7. We conjecture that the surjective linear map $\mathfrak{A}: H^{*}\left(X^{[n+1]}\right) \rightarrow$ $H^{*}\left(X^{[n]}\right)$ is a ring homomorphism for an arbitrary smooth quasi-projective surface $X$. By Lemma 4.5, this is equivalent to the Constant Conjecture in Wa].

Definition 4.8. Let $X$ be a smooth quasi-projective surface satisfying the Sproperty. We define the $F H$ ring $\mathcal{G}_{X}$ associated to $X$ to be the ring with a linear basis given by the symbols $\mathfrak{b}_{\rho_{X}}, \rho_{X} \in \mathcal{P}\left(S_{X}\right)$, with the multiplication given by

$$
\mathfrak{b}_{\rho_{X}} \cdot \mathfrak{b}_{\sigma_{X}}=\sum_{\nu_{X} \in \mathcal{P}\left(S_{X}\right)} a_{\rho_{X} \sigma_{X}}^{\nu_{X}} \mathfrak{b}_{\nu_{X}}
$$

where the structure constants $a_{\rho_{X} \sigma_{X}}^{\nu_{X}}$ come from (4.3).

For a smooth quasi-projective surface $X$ satisfying the S-property, define a linear map $\mathfrak{A}_{n}: \mathcal{G}_{X} \rightarrow H^{*}\left(X^{[n]}\right)$ by sending $\mathfrak{b}_{\rho_{X}}$ to $\mathfrak{b}_{\rho_{X}}(n)$. By the definition of $\mathcal{G}_{X}$ and Theorem $4.3 \mathfrak{A}_{n}$ is a surjective ring homomorphism. We can illustrate Theorem 4.3 in terms of the following commutative diagram of surjective ring homomorphisms:

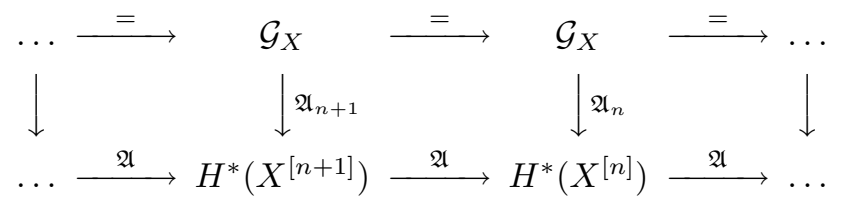

Next, we study the structure of the FH ring $\mathcal{G}_{X}$. For fixed $r \geq 1$ and $c \in S_{X}$, we use $\mathfrak{b}_{r, c}$ to denote $\mathfrak{b}_{\rho_{X}}$, where $\rho_{X} \in \mathcal{P}\left(S_{X}\right)$ is defined by taking $\rho_{X}(c)$ to be the one-part partition $(r)$ and $\rho_{X}\left(c^{\prime}\right)$ to be empty for each $c^{\prime} \neq c$.

Theorem 4.9. Let $X$ be a smooth quasi-projective surface which satisfies the $S$ property. Then the $F H$ ring $\mathcal{G}_{X}$ is isomorphic to the tensor product $P \otimes E$, where $P$ is the polynomial algebra generated by $\mathfrak{b}_{r, c}, c \in S_{X} \cap H^{\operatorname{even}}(X), r \geq 1$, and $E$ is the exterior algebra generated by $\mathfrak{b}_{r, c}, c \in S_{X} \cap H^{\text {odd }}(X), r \geq 1$.

Proof. Let notations be as above. First of all, we see from Lemma 3.7 that the FH ring $\mathcal{G}_{X}$ is generated by the elements $\mathfrak{b}_{r, c}$ with $r \geq 1$ and $c \in S_{X}$. Also, note that $\mathcal{G}_{X}$ is super-commutative and $\mathfrak{b}_{r, c}^{2}=0$ for $c \in S_{X} \cap H^{\text {odd }}(X)$.

It remains to show that as $\rho=\left(r^{m_{r}(c)}\right)_{c \in S_{X}, r \geq 1}$ runs over $\mathcal{P}\left(S_{X}\right)$, the monomials $\prod_{c \in S_{X}, r \geq 1} \mathfrak{b}_{r, c}^{m_{r}(c)}$ are linearly independent in $\mathcal{G}_{X}$. Assume

$$
\sum_{i \in I} d_{i} \prod_{c \in S_{X}, r \geq 1} \mathfrak{b}_{r, c}^{m_{r}^{i}(c)}=0
$$

where $d_{i} \in \mathbb{Q}$ and $\rho_{i}=\left(r^{m_{r}^{i}(c)}\right)_{c \in S_{X}, r \geq 1}$ runs over a finite set $I$ of distinct elements in $\mathcal{P}\left(S_{X}\right)$. By (3.2), we have $\mathfrak{b}_{r, c}(n)=\mathbf{1}_{-\left(n-r-\delta_{c}\right)} \mathfrak{a}_{-\left(r+\delta_{c}\right)}(c)|0\rangle$, where $\delta_{c}=0$ if $c \neq 1_{X}$ and $\delta_{c}=1$ if $c=1_{X}$. So we conclude that

$$
\sum_{i \in I} d_{i} \prod_{c \in S_{X}, r \geq 1}\left(\mathbf{1}_{-\left(n-r-\delta_{c}\right)} \mathfrak{a}_{-\left(r+\delta_{c}\right)}(c)|0\rangle\right)^{m_{r}^{i}(c)}=\sum_{i \in I} d_{i} \prod_{c \in S_{X}, r \geq 1} \mathfrak{b}_{r, c}(n)^{m_{r}^{i}(c)}=0 .
$$

Since $H^{*}\left(X^{[n]}\right) \cong H^{*}\left(\bar{X}^{[n]}\right) / \mathcal{I}^{[n]}$, we see from Lemma 4.1 that

$$
\sum_{i \in I} d_{i} \cdot \prod_{\bar{c} \in S-S_{\mathcal{I}}, r \geq 1}\left(\mathbf{1}_{-\left(n-r-\delta_{\iota^{*} \tau}\right)} \mathfrak{a}_{-\left(r+\delta_{\iota^{*} \tau}\right)}(\bar{c})|0\rangle\right)^{m_{r}^{i}\left(\iota^{*} \bar{c}\right)}=w \in \mathcal{I}^{[n]}
$$


Take an integer $n$ large enough such that $n \geq n_{i} \stackrel{\text { def }}{=} \sum_{r, \bar{c} \in S-S_{\mathcal{I}}}\left(r+\delta_{\iota^{*} \bar{c}}\right) m_{r}^{i}\left(\iota^{*} \bar{c}\right)$ for all $i \in I$. By the Theorem 5.1 in LQW3, (4.6) can be rewritten as

$$
\sum_{i \in I} d_{i}\left(\mathbf{1}_{-\left(n-n_{i}\right)}\left(\prod_{\bar{c} \in S-S_{\mathcal{I}}, r \geq 1}\left(\mathfrak{a}_{-\left(r+\delta_{\iota} *_{\bar{c}}\right)}(\bar{c})\right)^{m_{r}^{i}\left(\iota^{*} \bar{c}\right)}\right)|0\rangle+w_{i}\right)=w \in \mathcal{I}^{[n]}
$$

where each $w_{i}$ is a universal finite linear combination of $\mathbf{1}_{-(n-m)} \prod_{p=1}^{N} \mathfrak{a}_{-m_{p}}\left(\gamma_{p}\right) \cdot|0\rangle$ with $m=\sum_{p=1}^{N} m_{p}<n_{i}$ and $\gamma_{p} \in S$ for every $p$. Write $w$ and every $w_{i}$ as linear combinations of the basis (3.3). Since $\left(S-S_{\mathcal{I}}\right) \cap S_{\mathcal{I}}=\emptyset$, we see from (4.7) that

$$
\sum_{i} d_{i} \cdot \mathbf{1}_{-\left(n-n_{i}\right)}\left(\prod_{\bar{c} \in S-S_{\mathcal{I}, r \geq 1}}\left(\mathfrak{a}_{-\left(r+\delta_{\iota^{*} \bar{\tau}}\right)}(\bar{c})\right)^{m_{r}^{i}\left(\iota^{*} \bar{c}\right)}\right)|0\rangle=0
$$

where $i$ satisfies $n_{i}=\max \left\{n_{j} \mid j \in I\right\}$. Since the partitions $\rho_{i}=\left(r^{m_{r}^{i}(c)}\right)_{c \in S_{X}, r \geq 1} \in$ $\mathcal{P}\left(S_{X}\right)$ are distinct, all the coefficients $d_{i}$ in 4.8 must be zero. By repeating the above argument, we see that $d_{i}=0$ for all $i \in I$.

Corollary 4.10. Let $X$ be a smooth quasi-projective surface which satisfies the $S$ property. Then for $n \geq 1$, the cohomology ring $H^{*}\left(X^{[n]}\right)$ is generated by the classes $\mathfrak{b}_{r, c}(n)$, where $1 \leq r \leq n$ and $c$ runs over a linear basis of $H^{*}(X)$.

Proof. Follows from Theorem 4.9] and the observation that the ring homomorphism $\mathfrak{A}_{n}$ in the commutative diagram (4.5) is surjective.

We remark that Theorem 4.4 provides a set of ring generators for $H^{*}\left(X^{[n]}\right)$. Therefore, Corollary 4.10 gives us a second set of ring generators for $H^{*}\left(X^{[n]}\right)$, which is parallel to the set of ring generators for $H^{*}\left(\bar{X}^{[n]}\right)$ found in LQW2.

\subsection{Examples of quasi-projective surfaces with the S-property.}

Example 4.11. Let $\bar{X}$ be a projective surface and let $X$ be the quasi-projective surface obtained from $X$ with a point removed. It is easy to see that this smooth quasi-projective surface $X$ satisfies the S-property.

Example 4.12. Let $\Gamma$ be a finite subgroup of $S L_{2}(\mathbb{C})$. Let $X$ be the minimal resolution of the simple singularity $\mathbb{C}^{2} / \Gamma$. It is known that this smooth quasiprojective surface $X$ satisfies the S-property. Moreover, $K_{X}=0$.

Example 4.13 (The cotangent bundle of a smooth projective curve). Consider the ruled surface $\bar{X}=\mathbb{P}\left(\mathcal{O}_{C}\left(-K_{C}\right) \oplus \mathcal{O}_{C}\right)$, where $C$ is a smooth projective curve. Let $\sigma$ be the section (to the projection $\bar{X} \rightarrow C$ ) corresponding to the natural surjection $\mathcal{O}_{C}\left(-K_{C}\right) \oplus \mathcal{O}_{C} \rightarrow \mathcal{O}_{C}\left(-K_{C}\right) \rightarrow 0$, and put $X=\bar{X}-\sigma$. Then, $X$ is the total space of the cotangent bundle of $C$, and $K_{X}=0$.

We claim that $X$ satisfies the S-property. In fact, the following general statement is true. Let $\bar{X}=\mathbb{P}\left(\mathcal{L}_{1} \oplus \mathcal{L}_{2}\right)$, where $\mathcal{L}_{1}$ and $\mathcal{L}_{2}$ are two invertible sheaves over $C$. Let $\sigma$ (resp. $\sigma^{\prime}$ ) be the section of $\bar{X} \rightarrow C$ corresponding to the natural surjection $\mathcal{L}_{1} \oplus \mathcal{L}_{2} \rightarrow \mathcal{L}_{1} \rightarrow 0$ (resp. $\quad \mathcal{L}_{1} \oplus \mathcal{L}_{2} \rightarrow \mathcal{L}_{2} \rightarrow 0$ ). Put $X=\bar{X}-\sigma$. Then $X$ satisfies the $\mathrm{S}$-property. To see this, let $X^{\prime}=\bar{X}-\sigma^{\prime}$, and notice that $X$ and $X^{\prime}$ are affine bundles over $C$. Hence $X$ is homotopic to $C$, and $H^{i}(X) \cong H^{i}(C)$ for every $i$. Therefore, to verify the S-property of $X$, it remains to verify the surjectivities of the induced homomorphisms $r_{i}: H^{i}(\bar{X}) \rightarrow H^{i}(X)$ for $i=1,2$. Consider the relative 
cohomology group $H^{2}(\bar{X}, X)$. By the excision theorem, we obtain $H^{2}(\bar{X}, X) \cong$ $H^{2}\left(\bar{X}-\sigma^{\prime}, X-\sigma^{\prime}\right)=H^{2}\left(X^{\prime}, X^{\prime}-\sigma\right)$. By the Thom isomorphism, since $X^{\prime}$ is an affine bundle over $C$ with $\sigma$ being the zero section, we have $H^{2}\left(X^{\prime}, X^{\prime}-\sigma\right) \cong$ $H^{0}(C) \cong \mathbb{Q}$. Hence $H^{2}(\bar{X}, X) \cong \mathbb{Q}$. Now consider the exact sequence

$$
H^{1}(\bar{X}) \stackrel{r_{1}}{\longrightarrow} H^{1}(X) \stackrel{\delta}{\longrightarrow} H^{2}(\bar{X}, X) \longrightarrow H^{2}(\bar{X}) \stackrel{r_{2}}{\longrightarrow} H^{2}(X) .
$$

Since $H^{2}(X) \cong H^{2}(C) \cong \mathbb{Q}$ and $H^{2}(\bar{X}) \cong \mathbb{Q} \oplus \mathbb{Q}$, we conclude that the map $\delta$ must be zero and the map $r_{2}$ must be surjective. Therefore, $r_{1}$ is also surjective.

\section{ORBIFOLD COHOMOLOGY RINGS OF SYMMETRIC PRODUCTS}

The orbifold cohomology ring of an orbifold was introduced in CR. Given an even-dimensional compact complex manifold $X$, the orbifold cohomology rings $H_{\mathrm{orb}}^{*}\left(X^{n} / S_{n}\right)$ of symmetric products $X^{n} / S_{n}$ were studied in [FG], QW], Uri] (also cf. [LS2, [Ru1]). The axiomatic approach in QW] is self-contained within the framework of the symmetric products, while it is parallel to the study of the cohomology rings of Hilbert schemes $X^{[n]}$ when $X$ is a smooth projective surface. The results obtained in the previous sections for the cohomology rings of $X^{[n]}$ when $X$ is a smooth quasi-projective surface are built on the works LQW3, LQW4 (also cf. [Lehn], [LQW1]). As observed in [QW], all the results in [LQW3], LQW4] admit exact counterparts in the orbifold cohomology rings of symmetric products. This allows us to readily obtain the results on $H_{\text {orb }}^{*}\left(X^{n} / S_{n}\right)$ when $X$ is an evendimensional noncompact complex manifold, which are the counterparts of those on the cohomology rings of $X^{[n]}$ when $X$ is a smooth quasi-projective surface.

In this section, we will formulate and sketch these analogous results for the orbifold cohomology rings $H_{\mathrm{orb}}^{*}\left(X^{n} / S_{n}\right)$ when $X$ is noncompact. We will not repeat the proofs for these analogous results since the proofs are the same as in the Hilbert scheme setup. For notational simplicity, we will assume below that $X$ is a smooth quasi-projective surface which satisfies the S-property (the assumption on the dimension of $X$ can be relaxed without extra difficulty). We will use the results of QW] freely and refer the reader to loc. cit. for details.

Let $\bar{X}$ be a smooth projective surface which contains $X$ such that the pullback map $\iota^{*}: H^{*}(\bar{X}) \rightarrow H^{*}(X)$ is surjective, where $\iota: X \rightarrow \bar{X}$ is the inclusion map. Recall from Section 5.1 in $[\mathrm{QW}$ that there exists a family of ring products, denoted by $\circ_{t}$, on $H_{\text {orb }}^{*}\left(\bar{X}^{n} / S_{n}\right)$ depending on a rational (or complex) parameter $t$. When $t=1$, this coincides with the original definition of orbifold product $[\mathrm{CR}$ and when $t=-1$, this coincides with the modified product in [LS2], [FG], Uri]. Put

$$
\mathcal{F}_{\bar{X}}=\bigoplus_{n=0}^{\infty} H_{\text {orb }}^{*}\left(\bar{X}^{n} / S_{n}\right)
$$

We can define the Heisenberg algebra acting irreducibly on $\mathcal{F}_{\bar{X}}$ with linear basis ${ }^{t} \mathfrak{p}_{n}(\gamma)$, where $n \in \mathbb{Z}$ and $\gamma \in H^{*}(\bar{X})$. The elements in this linear basis satisfy the commutation relation (cf. Sections 3.2 and 5.2 in $[\mathrm{QW}$ )

$$
\left[{ }^{t} \mathfrak{p}_{m}(\alpha),{ }^{t} \mathfrak{p}_{n}(\beta)\right]=t^{1 / 3} m \delta_{m,-n} \int_{\bar{X}}(\alpha \beta) \cdot \operatorname{Id}_{\mathcal{F}_{\bar{X}}} .
$$

When $t=-1$, this matches with the commutation relation of the Heisenberg algebra associated to the Hilbert schemes (compare with (2.1)). 
The above definitions of the Fock space and of Heisenberg algebra readily extend to $X$. Set $\mathcal{F}_{X}=\bigoplus_{n=0}^{\infty} H_{\text {orb }}^{*}\left(X^{n} / S_{n}\right)$. The creation operators ${ }^{t} \mathfrak{p}_{-n}(\gamma)$, where $n>0$ and $\gamma \in H^{*}(X)$, are defined in the same way as before, while the annihilation operators ${ }^{t} \mathfrak{p}_{n}(\gamma)$, where $n>0$, are modelled on $\gamma \in H_{c}^{*}(X)$.

The definition in Sections 3.4 and 5.2 of QW] of the cohomology classes $\eta_{n}(\alpha)$ and $O^{k}(\alpha, n)$ in $H_{\text {orb }}^{*}\left(X^{n} / S_{n}\right)$ remain to be valid for $X$ quasi-projective without any change. We further define $O_{k}(\alpha, n)=O^{k}(\alpha, n) / k$ !. In the same way, we define the operator ${ }^{t} \mathfrak{O}^{k}(\alpha)$ (resp. $\left.{ }^{t} \mathfrak{O}_{k}(\alpha)\right)$ in $\operatorname{End}\left(\mathcal{F}_{X}\right)$ to be the orbifold product $o_{t}$ with the class $O^{k}(\alpha, n)$ (resp. with the class $\left.O_{k}(\alpha, n)\right)$ in $H_{\text {orb }}^{*}\left(X^{n} / S_{n}\right)$ for every $n \geq 0$.

The inclusion map $\iota: X \rightarrow \bar{X}$ induces an evident surjective ring homomorphism $\jmath_{n}^{*}: H_{\text {orb }}^{*}\left(\bar{X}^{n} / S_{n}\right) \rightarrow H_{\text {orb }}^{*}\left(X^{n} / S_{n}\right)$ (note that $\jmath_{1}^{*}=\iota^{*}$ is surjective by assumption). We have the analogue of Lemma 4.1, namely,

$$
J_{n}^{*}\left({ }^{t} \mathfrak{p}_{-n_{1}}\left(\bar{\alpha}_{1}\right) \cdots{ }^{t} \mathfrak{p}_{-n_{s}}\left(\bar{\alpha}_{s}\right)|0\rangle\right)={ }^{t} \mathfrak{p}_{-n_{1}}\left(\iota^{*} \bar{\alpha}_{1}\right) \cdots{ }^{t} \mathfrak{p}_{-n_{s}}\left(\iota^{*} \bar{\alpha}_{s}\right)|0\rangle,
$$

where $n_{1}, \ldots, n_{s}>0, n_{1}+\ldots+n_{s}=n$, and $\bar{\alpha}_{i} \in H^{*}(\bar{X})$. Here $|0\rangle$ denotes $1 \in H_{\text {orb }}^{*}(p t)$ as usual. Given $\bar{\alpha} \in H^{*}(\bar{X})$, we have by construction

$$
\jmath_{n}^{*}\left(O_{k}(\bar{\alpha}, n)\right)=O_{k}\left(\iota^{*} \bar{\alpha}, n\right) .
$$

The following is the counterpart of Theorem 4.4.

Proposition 5.1. Let $X$ be a smooth quasi-projective surface embedded in a smooth projective surface $\bar{X}$ such that the induced map $H^{*}(\bar{X}) \rightarrow H^{*}(X)$ is surjective. Then, the cohomology classes $O_{k}(\alpha, n)$, as $0 \leq k<n$ and $\alpha$ runs over a linear basis of $H^{*}(X)$, form a set of ring generators of $H_{\mathrm{orb}}^{*}\left(X^{n} / S_{n}\right)$.

Apparently, we can introduce a linear basis $\mathfrak{q}_{\rho_{X}}(n)$ of $H_{\text {orb }}^{*}\left(X^{n} / S_{n}\right)$ in terms of the Heisenberg generators ${ }^{t} \mathfrak{p}_{n}(\alpha)$, where $\rho_{X} \in \mathcal{P}\left(S_{X}\right)$ such that $\left\|\rho_{X}\right\|+\ell\left(\rho_{X}\left(1_{X}\right)\right) \leq$ $n$, which is the counterpart of the linear basis $\mathfrak{b}_{\rho_{X}}$ for $H^{*}\left(X^{[n]}\right)$. We write

$$
\mathfrak{q}_{\rho_{X}}(n) \circ_{t} \mathfrak{q}_{\sigma_{X}}(n)=\sum_{\nu_{X}} p_{\rho_{X} \sigma_{X}}^{\nu_{X}}(n) \mathfrak{q}_{\nu_{X}}(n)
$$

where $p_{\rho_{X} \sigma_{X}}^{\nu_{X}}(n)$ denotes the structure constants for the orbifold product. The following proposition is the counterpart of Theorem 4.3

Proposition 5.2. Let $X$ be a smooth quasi-projective surface which satisfies the $S$-property. Then all the structure constants $p_{\rho_{X} \sigma_{X}}^{\nu_{X}}(n)$ are independent of $n$.

Remark 5.3. Thanks to Proposition 5.2, we will denote $p_{\rho_{X}}^{\nu_{X}} \sigma_{X}(n)$ simply by $p_{\rho_{X} \sigma_{X}}^{\nu_{X}}$. It follows from Proposition 5.2 that we can introduce a universal ring $\mathcal{U}_{X}$ (referred to again as the $\mathrm{FH}$ ring) with a linear basis given by the symbols $\mathfrak{q}_{\rho_{X}}$ and multiplication given by $\mathfrak{q}_{\rho_{X}} \circ \mathfrak{q}_{\sigma_{X}}=\sum_{\nu_{X}} p_{\rho_{X} \sigma_{X}}^{\nu_{X}} \mathfrak{q}_{\nu_{X}}$. This $\mathrm{FH}$ ring $\mathcal{U}_{X}$ governs the orbifold cohomology ring $\left(H_{\text {orb }}^{*}\left(X^{n} / S_{n}\right), \circ_{t}\right)$ for a fixed smooth quasi-projective surface with the S-property and for every $n$. Similarly, we have a second set of ring generators for $H_{\mathrm{orb}}^{*}\left(X^{n} / S_{n}\right)$ which is the counterpart of Corollary 4.10

We introduce the linear isomorphisms $\Theta: \mathcal{F}_{X} \rightarrow \mathbb{H}_{X}$ and $\Theta_{n}: H_{\text {orb }}^{*}\left(X^{n} / S_{n}\right) \rightarrow$ $H^{*}\left(X^{[n]}\right)$ by sending ${ }^{t} \mathfrak{p}_{-n_{1}}\left(\alpha_{1}\right) \cdots{ }^{t} \mathfrak{p}_{-n_{s}}\left(\alpha_{s}\right)|0\rangle$ to $\mathfrak{a}_{-n_{1}}\left(\alpha_{1}\right) \cdots \mathfrak{a}_{-n_{s}}\left(\alpha_{s}\right)|0\rangle$. Similarly, we define the linear isomorphisms $\bar{\Theta}: \mathcal{F}_{\bar{X}} \rightarrow \mathbb{H}_{\bar{X}}$ and $\bar{\Theta}_{n}: H_{\text {orb }}^{*}\left(\bar{X}^{n} / S_{n}\right) \rightarrow$ 
$H^{*}\left(\bar{X}^{n} / S_{n}\right)$. We have the following commutative diagram by definitions:

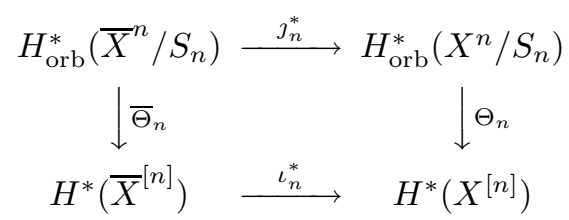

Theorem 5.4. Let $X$ be a smooth quasi-projective surface with the S-property and numerically trivial canonical class. Then the linear map $\Theta_{n}: H_{\mathrm{orb}}^{*}\left(X^{n} / S_{n}\right) \rightarrow$ $H^{*}\left(X^{[n]}\right)$ is a ring isomorphism, if we use the product $0_{-1}$ on $H_{\mathrm{orb}}^{*}\left(X^{n} / S_{n}\right)$.

Proof. Set $t=-1$. However, for notational convenience, we will keep writing ${ }^{t_{\mathfrak{p}}}$ instead of ${ }^{-1} \mathfrak{p}$, etc. The axiomatic approach in $[\mathrm{QW}$ shows that the operator $\mathfrak{O}_{k}(\bar{\alpha}) \in \operatorname{End}\left(\mathcal{F}_{\bar{X}}\right)$, where $\bar{\alpha} \in H^{*}(\bar{X})$, is equal to

$$
-\sum_{\ell(\lambda)=k+2,|\lambda|=0} \frac{1}{\lambda^{!}}{ }^{t} \mathfrak{p}_{\lambda}\left(\tau_{*} \bar{\alpha}\right)+\sum_{\ell(\lambda)=k,|\lambda|=0} \frac{s(\lambda)-2}{24 \lambda^{!}}{ }^{t} \mathfrak{p}_{\lambda}\left(\tau_{*}\left(e_{\bar{X}} \bar{\alpha}\right)\right),
$$

where $e_{\bar{X}}$ is the Euler class of $\bar{X}$. We remark that no term in (5.6) involves the canonical class $K_{\bar{X}}$ of $\bar{X}$ in contrast to the formula for $\mathfrak{G}_{k}(\bar{\alpha})$ in Theorem 2.4

Let $\epsilon \in\left\{K_{\bar{X}},\left(K_{\bar{X}}\right)^{2}\right\}, \ell(\lambda)=k+2-|\epsilon| / 2$ and $|\lambda|=0$. Let $\bar{\alpha}_{i} \in H^{*}(\bar{X}), n_{i}>0$ $(i=1, \ldots, s)$, and $n_{1}+\ldots+n_{s}=n$. Applying the analogues of Lemma 2.1 (i) to ${ }^{t} \mathfrak{p}$, we see that the expression ${ }^{t} \mathfrak{p}_{\lambda}\left(\tau_{*}(\epsilon \bar{\alpha}){ }^{t} \mathfrak{p}_{-n_{1}}\left(\bar{\alpha}_{1}\right) \cdots{ }^{t} \mathfrak{p}_{-n_{s}}\left(\bar{\alpha}_{s}\right)|0\rangle\right.$ is a linear combination of Heisenberg monomials of the form

$$
{ }^{t} \mathfrak{p}_{-n_{1}^{\prime}}\left(\bar{\alpha}_{1}^{\prime}\right) \cdots{ }^{t} \mathfrak{p}_{-n_{u}^{\prime}}\left(\bar{\alpha}_{u}^{\prime}\right)\left({ }^{t} \mathfrak{p}_{-n_{u+1}^{\prime}} \ldots{ }^{t} \mathfrak{p}_{-n_{u+v}^{\prime}}\right)\left(\tau_{v *}\left(K_{\bar{X}} \bar{\alpha}^{\prime}\right)\right)|0\rangle,
$$

where $v>0, n_{1}^{\prime}, \ldots, n_{u+v}^{\prime}>0$, and $n_{1}^{\prime}+\ldots+n_{u+v}^{\prime}=n$. From the proof of Lemma 2.5. $\tau_{v *}\left(K_{\bar{X}} \bar{\alpha}^{\prime}\right)=\sum_{j}\left(K_{\bar{X}} \bar{\alpha}_{1, j}^{\prime \prime}\right) \otimes \bar{\alpha}_{2, j}^{\prime \prime} \otimes \cdots \otimes \bar{\alpha}_{v, j}^{\prime \prime}$. Since $\iota^{*} K_{\bar{X}}=K_{X}=0$ by assumption, $\iota^{*}\left(K_{\bar{X}} \bar{\alpha}_{1, j}^{\prime \prime}\right)=0$. By Lemma 4.1, we conclude that

$$
\iota_{n}^{*} \bar{\Theta}_{n}\left({ }^{t} \mathfrak{p}_{\lambda}\left(\tau_{*}(\epsilon \bar{\alpha}){ }^{t} \mathfrak{p}_{-n_{1}}\left(\bar{\alpha}_{1}\right) \cdots{ }^{t} \mathfrak{p}_{-n_{s}}\left(\bar{\alpha}_{s}\right)|0\rangle\right)=0 .\right.
$$

Now consider a given Heisenberg monomial $A=\mathfrak{a}_{-n_{1}}\left(\alpha_{1}\right) \cdots \mathfrak{a}_{-n_{s}}\left(\alpha_{s}\right)|0\rangle \in$ $H^{*}\left(X^{[n]}\right)$, where $\alpha_{i} \in H^{*}(X), n_{i}>0(i=1, \ldots, s)$, and $n_{1}+\ldots+n_{s}=n$. Fix $\bar{\alpha}_{i} \in H^{*}(\bar{X})$ such that $\iota^{*}\left(\bar{\alpha}_{i}\right)=\alpha_{i}$. Put $P={ }^{t} \mathfrak{p}_{-n_{1}}\left(\alpha_{1}\right) \cdots{ }^{t} \mathfrak{p}_{-n_{s}}\left(\alpha_{s}\right)|0\rangle$. Given $\alpha \in H^{*}(X)$, we choose $\bar{\alpha} \in H^{*}(\bar{X})$ such that $\iota^{*}(\bar{\alpha})=\alpha$. We have

$$
\begin{aligned}
\Theta_{n}\left(O_{k}(\alpha, n) \circ_{t} P\right) & =\Theta_{n} J_{n}^{*}\left(O_{k}(\bar{\alpha}, n) \circ_{t}{ }^{t} \mathfrak{p}_{-n_{1}}\left(\bar{\alpha}_{1}\right) \ldots{ }^{t} \mathfrak{p}_{-n_{s}}\left(\bar{\alpha}_{s}\right)|0\rangle\right) \\
& =\Theta_{n} J_{n}^{*}\left(\mathfrak{O}_{k}(\bar{\alpha}){ }^{t} \mathfrak{p}_{-n_{1}}\left(\bar{\alpha}_{1}\right) \cdots{ }^{t} \mathfrak{p}_{-n_{s}}\left(\bar{\alpha}_{s}\right)|0\rangle\right) \\
& =\iota_{n}^{*} \bar{\Theta}_{n}\left(\mathfrak{O}_{k}(\bar{\alpha}){ }^{t} \mathfrak{p}_{-n_{1}}\left(\bar{\alpha}_{1}\right) \cdots{ }^{t} \mathfrak{p}_{-n_{s}}\left(\bar{\alpha}_{s}\right)|0\rangle\right),
\end{aligned}
$$

where we have used (5.5) and the fact that $\jmath_{n}^{*}$ is a ring homomorphism. By (5.6), (5.7) and Theorem 2.4, we get $\iota_{n}^{*} \bar{\Theta}_{n}\left(\mathfrak{O}_{k}(\bar{\alpha}){ }^{t} \mathfrak{p}_{-n_{1}}\left(\bar{\alpha}_{1}\right) \cdots{ }^{t} \mathfrak{p}_{-n_{s}}\left(\bar{\alpha}_{s}\right)|0\rangle\right)=$ $\iota_{n}^{*}\left(\mathfrak{G}_{k}(\bar{\alpha}) \mathfrak{a}_{-n_{1}}\left(\bar{\alpha}_{1}\right) \cdots \mathfrak{a}_{-n_{s}}\left(\bar{\alpha}_{s}\right)|0\rangle\right)$. Since $\iota_{n}^{*}$ is a ring homomorphism, we obtain

$$
\begin{aligned}
& \Theta_{n}\left(O_{k}(\alpha, n) \circ_{t} P\right)=\iota_{n}^{*}\left(\mathfrak{G}_{k}(\bar{\alpha}) \mathfrak{a}_{-n_{1}}\left(\bar{\alpha}_{1}\right) \cdots \mathfrak{a}_{-n_{s}}\left(\bar{\alpha}_{s}\right)|0\rangle\right) \\
= & \iota_{n}^{*}\left(G_{k}(\bar{\alpha}, n) \cdot \mathfrak{a}_{-n_{1}}\left(\bar{\alpha}_{1}\right) \cdots \mathfrak{a}_{-n_{s}}\left(\bar{\alpha}_{s}\right)|0\rangle\right)=G_{k}(\alpha, n) \cdot A,
\end{aligned}
$$

noting that $\iota_{n}^{*} G_{k}(\bar{\alpha}, n)=G_{k}(\alpha, n)$ by definition. Letting $P$ be the unit of the cohomology ring $H_{\mathrm{orb}}^{*}\left(X^{n} / S_{n}\right)$, we obtain from (5.8) that $\Theta_{n}\left(O_{k}(\alpha, n)\right)=G_{k}(\alpha, n)$. Now our theorem follows from (5.8), Theorem 4.4 and Proposition 5.1 
Corollary 5.5. With the same assumptions as in Theorem 5.4, the structure constant $a_{\rho_{X} \sigma_{X}}^{\nu_{X}}$ in (4.3) and the structure constant $p_{\rho_{X} \sigma_{X}}^{\nu_{X}}$ in (5.4) are equal.

Proof. Follows immediately from (4.3), (5.4) and Theorem 5.4.

Remark 5.6. The analogue to Theorem 5.4 for smooth projective surfaces with numerically trivial canonical class was established in [LS2], [FG], [Uri] and QW]. As pointed out in $[\mathrm{QW}$, when the coefficient is taken to be $\mathbb{C}$ instead of $\mathbb{Q}$, there exist explicit ring isomorphisms among the rings $\left(H_{\text {orb }}^{*}\left(X^{n} / S_{n} ; \mathbb{C}\right), o_{t}\right)$ for nonzero $t$. In particular, when combined with Theorem 5.4 this implies that the cohomology ring $H^{*}\left(X^{[n]} ; \mathbb{C}\right)$ is isomorphic to the original orbifold cohomology ring $\left(H_{\text {orb }}^{*}\left(X^{n} / S_{n} ; \mathbb{C}\right), \circ_{1}\right)$ for smooth quasi-projective surfaces $X$ satisfying the $\mathrm{S}$ property and having numerically trivial canonical classes. This further supports Ruan's conjecture in Ru1, Ru2]. Finally, we notice that there are many examples of smooth quasi-projective surfaces with the S-property and numerically trivial canonical classes, including Example 4.12 and Example4.13.

\section{ACKNOWLEDGMENT}

We thank M. Lehn and Y. Ruan for helpful discussions.

\section{REFERENCES}

[CR] W. Chen, Y. Ruan, A new cohomology theory for orbifold, arXiv:math.AG/0004129.

[ES] G. Ellingsrud, S. Strømme, Towards the Chow ring of the Hilbert scheme of $\mathbb{P}^{2}$, J. Reine Angew. Math. 441 (1993), 33-44. MR 94i:14004

[FG] B. Fantechi, L. Göttsche, Orbifold cohomology for global quotients, Duke Math. J. 117 (2003), 197-227.

[FH] H. Farahat, G. Higman, The centres of symmetric group rings, Proc. Roy. Soc. (A) 250 (1959), 212-221. MR 21:2697

[Got] L. Göttsche, The Betti numbers of the Hilbert scheme of points on a smooth projective surface, Math. Ann. 286 (1990), 193-207. MR 91h:14007

[Gro] I. Grojnowski, Instantons and affine algebras I: the Hilbert scheme and vertex operators, Math. Res. Lett. 3 (1996), 275-291. MR 97f:14041

[Lehn] M. Lehn, Chern classes of tautological sheaves on Hilbert schemes of points on surfaces, Invent. Math. 136 (1999), 157-207. MR 2000h:14003

[LS1] M. Lehn, C. Sorger, Symmetric groups and the cup product on the cohomology of Hilbert schemes, Duke Math. J. 110 (2001), 345-357. MR 2002i:14004

[LS2] M. Lehn, C. Sorger, The cup product of the Hilbert scheme for K3 surfaces, Invent. Math. 152 (2003) 305-329.

[LQW1] W.-P. Li, Z. Qin, W. Wang, Vertex algebras and the cohomology ring structure of Hilbert schemes of points on surfaces, Math. Ann. 324 (2002), no. 1, 105-133. MR 2003h:14009

[LQW2] W.-P. Li, Z. Qin, W. Wang, Generators for the cohomology ring of Hilbert schemes of points on surfaces, Intern. Math. Res. Notices 20 (2001), 1057-1074. MR 2002j:14003

[LQW3] W.-P. Li, Z. Qin, W. Wang, Stability of the cohomology rings of Hilbert schemes of points on surfaces, J. Reine Angew. Math. 554 (2003), 217-234.

[LQW4] W.-P. Li, Z. Qin, W. Wang, Hilbert schemes and $\mathcal{W}$ algebras, Intern. Math. Res. Notices 27 (2002), 1427-1456. MR 2003d:17033

[Na1] H. Nakajima, Heisenberg algebra and Hilbert schemes of points on projective surfaces, Ann. Math. 145 (1997), 379-388. MR 98h:14006

[Na2] H. Nakajima, Lectures on Hilbert schemes of points on surfaces, Univ. Lect. Ser. 18, Amer. Math. Soc. (1999). MR 2001b:14007

[Ru1] Y. Ruan, Stringy geometry and topology of orbifolds, Symposium in Honor of C. H. Clemens (Salt Lake City, UT, 2000), 187-233, Contemp. Math., 312, Amer. Math. Soc., Providence, RI, 2002.

[Ru2] Y. Ruan, Cohomology ring of crepant resolutions of orbifolds, Preprint, arXiv:ath.AG/0108195. 
[QW] Z. Qin, W. Wang, Hilbert schemes and symmetric products: a dictionary, Orbifolds in Mathematics and Physics (Madison, WI, 2001), 233-257, Contemp. Math., 310, Amer. Math. Soc., Providence, RI, 2002.

[Uri] B. Uribe, Orbifold cohomology of the symmetric product, Preprint, math.AT/0109125.

[VW] C. Vafa, E. Witten, A strong coupling test of $S$-duality, Nucl. Phys. B 431 (1994), 3-77. MR 95k:81138

[Vas] E. Vasserot, Sur l'anneau de cohomologie du schéma de Hilbert de $\mathbb{C}^{2}, \mathrm{C}$. R. Acad. Sci. Paris, Sér. I Math. 332 (2001), 7-12. MR 2001k:14012

[Wa] W. Wang, The Farahat-Higman ring of wreath products and Hilbert schemes, Preprint 2002, arXiv:math.QA/0205071.

Department of Mathematics, Hong Kong University of Science and Technology, Clear Water Bay, Kowloon, Hong Kong

E-mail address: mawpli@ust.hk

Department of Mathematics, University of Missouri, Columbia, Missouri 65211

E-mail address: zq@math.missouri.edu

Department of Mathematics, University of Virginia, Charlottesville, Virginia 22904

E-mail address: ww9c@virginia.edu 